\title{
Predicting Hydrogen Storage in MOFs via Machine Learning
}

\author{
Alauddin Ahmed $^{+}$and Donald J. Siegel ${ }^{+,, \Delta, B, *}$ \\ ${ }^{\dagger}$ Mechanical Engineering Department, ${ }^{\S}$ Materials Science \& Engineering, ${ }^{\triangle}$ Applied Physics Program, and ${ }^{B}$ University of Michigan En- \\ ergy Institute, University of Michigan, Ann Arbor, MI 48109, United States
}

\begin{abstract}
The $\mathrm{H}_{2}$ storage capacities of a diverse set of 918,734 metal-organic frameworks (MOFs) sourced from 19 databases is predicted via machine learning $(\mathrm{ML})$. Using only 7 structural features as input, ML identifies 8,282 MOFs with the potential to exceed the capacities of state-of-the-art materials under physisorptive conditions. The identified MOFs are predominantly hypothetical compounds having low densities $\left(<0.31 \mathrm{~g} \mathrm{~cm}^{-3}\right)$ in combination with high surface areas $\left(>5,300 \mathrm{~m}^{2} \mathrm{~g}^{-1}\right)$, void fractions $(\sim 0.90)$, and pore volumes $\left(>3.3 \mathrm{~cm}^{3} \mathrm{~g}^{-1}\right)$. In addition, the relative importance of the input features are characterized, and dependencies on the ML algorithm and training set size are quantified. The single most important features for predicting $\mathrm{H}_{2}$ uptake are pore volume (for gravimetric capacity) and void fraction (for volumetric capacity). The ML models are available for use via the web, allowing for rapid and accurate predictions of usable hydrogen capacities for MOFs with only minimal structural data as input; for the simplest models only a single input feature is required.
\end{abstract}

KEYWORDS: Energy storage fuel cells; MOFs; hydrogen storage; machine learning

\section{INTRODUCTION}

Hydrogen $\left(\mathrm{H}_{2}\right)$ is considered to be a future automotive fuel. ${ }^{1-6}$ This potential reflects its high specific energy compared to competing fuels such as natural gas and gasoline, and the ability of $\mathrm{H}_{2}$ to be produced renewably and consumed without $\mathrm{CO}_{2}$ emissions. ${ }^{2,7} \mathrm{Nev}-$ ertheless, the adoption of hydrogen in mobile applications such as fuel cell (FC) vehicles has been limited by its low volumetric energy density. ${ }^{2,6,7}$ Consequently, the design of low-cost $\mathrm{H}_{2}$ storage systems that overcome these volumetric limitations has been the focus of recent research. ${ }^{4,8-12}$ At present, FC vehicles employ storage systems based on gaseous $\mathrm{H}_{2}$ compressed to pressures up to 700 bar. ${ }^{13}$ This approach is costly and can incur limitations in driving range. ${ }^{7,11,13,14}$

Storage based on adsorption in porous hosts is an alternative to high-pressure compression..$^{15}$ Due to their high gravimetric densities, fast kinetics, and reversibility, metal-organic frameworks (MOFs) have emerged as one of the most promising classes of hydrogen sorbents. ${ }^{2,7}$ MOFs are crystalline materials formed by the self-assembly of inorganic metal clusters and organic linkers. ${ }^{16-22}$ By virtue of their building-block structure and the large number of potential components, the number of MOFs is potentially limitless. ${ }^{21-}$ ${ }^{25}$ Further modifications to MOF chemistry can be achieved by introducing functional groups, substituting different metals, and by mixing metals and/or linkers. ${ }^{26-28}$

Despite these many possibilities, a relatively small fraction of MOFs have been synthesized. ${ }^{29,30}$ While the crystal structures of these 'real' MOFs are available in the Cambridge Structural Database (CSD), ${ }^{29,30}$ many exhibit disorder, missing atoms, or have negligible porosity; consequently, these materials are not immediately amenable to assessment via computational modeling. ${ }^{29,31-35}$

One way to bypass these complications is through computational design. To date, nearly a million 'hypothetical' MOFs have been reported, 1,36,45,46,37-44 and it is reasonable to expect that many more materials will be proposed. ${ }^{4-51}$ High-throughput screening using Grand Canonical Monte Carlo (GCMC $)^{52-56}$ has been successful in identifying promising candidates with superior gas storage capacities on sub-sets of these catalogs. ${ }^{36,38,39,46,50,57-60}$ Nevertheless, given the large number of possibilities, a systematic search across all of these materials is challenging even with high-throughput techniques. ${ }^{1,61}$ Furthermore, differences in the implementation (i.e., use of different temperature/pressure conditions or interatomic potentials) can complicate comparisons between screening studies. Thus, more efficient and consistent screening approaches are desirable for predicting the gas storage properties of MOFs in existing and future databases.

Machine learning (ML) could provide a path forward. ${ }^{62-65}$ For ML to be helpful, access to high-quality training data is essential. Unfortunately, training on experimental $\mathrm{H}_{2}$ storage data in MOFs is non-trivial: ${ }^{1,2,6,66-68}$ experimental uptake data is generally restricted to a relatively small number of MOFs, and can depend sensitively upon the experimental conditions and the purity of the sample. ${ }^{2,67,69} \mathrm{Em}-$ ploying a dataset based on a consistent set of computational predictions may be a better choice..$^{62,63}$

Earlier work has demonstrated that accurate isotherms for $\mathrm{H}_{2}$ uptake in MOFs can be predicted using the pseudo-Feynman-Hibbs potential (to describe $\mathrm{H}_{2}$ ) combined with general interatomic potentials to describe the MOF. ${ }^{1,2,6,70}$ This approach was used to screen a database of 5,309 real MOFs, from which IRMOF-20 was identified and experimentally demonstrated to have a favorable balance of high gravimetric and volumetric $\mathrm{H}_{2}$ density. ${ }^{2}$ In a follow-on study a larger database of 495,305 MOFs was compiled from several publicly-available databases (see Table S1 for details). ${ }^{1,29,31,33,36-40,45}$ Following a pre-screen based on crystallographic properties and empirical correlations, the $\mathrm{H}_{2}$ capacities of a subset of 43,777 MOFs were evaluated using GCMC. Three additional MOFs - SNU-70, UMCM-9, and PCN-610/NU-100 - were identified and shown experimentally to out-perform the leading MOF candidate, IRMOF$20 .{ }^{1}$ 
Table 1: Summary of recent studies that use machine learning (ML) to predict $\mathrm{H}_{2}$ adsorption in MOFs. $\rho_{\text {crys, }}$ vf, vsa, mpd, lcd represent single crystal density, void fraction, volumetric surface area, maximum pore diameter, and largest cavity diameter, respectively. $R^{2}$, AUE, and RMSE represent the coefficient of determination, average unsigned error, and root-mean-square error, respectively.

\begin{tabular}{|c|c|c|c|c|}
\hline Study & ML Features & ML Method & Properties Predicted & Accuracy \\
\hline $\begin{array}{l}\text { Anderson et } \\
\text { al. }(2019)^{45}\end{array}$ & $\begin{array}{l}\text { Epsilon, temperature, pressure, } \rho_{\text {crys }} \\
\text { vf, vsa, mpd, lcd, alchemical cate- } \\
\text { cholate site density, unit cell volume }\end{array}$ & Neural network ${ }^{77}$ & $\begin{array}{l}\text { Total volumetric } \mathrm{H}_{2} \text { for pres- } \\
\text { sures } 0.1,1,5,35,65 \text {, and } 100 \\
\text { bar at } 77,160 \text {, and } 295 \mathrm{~K}\end{array}$ & AUE $=0.75-2.93 \mathrm{~g}-\mathrm{H}_{2} \mathrm{~L}^{-1}$ \\
\hline $\begin{array}{l}\text { Bucior et al. } \\
(2019)^{82}\end{array}$ & $\begin{array}{l}\text { Energetics of MOF-guest interac- } \\
\text { tions }\end{array}$ & $\begin{array}{l}\text { Multilinear regression } \\
\text { with } \mathrm{LASSO}^{77}\end{array}$ & $\begin{array}{l}\text { Deliverable } \mathrm{H}_{2} \text { storage capac- } \\
\text { ity between } 2-100 \text { bar at } 77 \mathrm{~K}\end{array}$ & $\begin{array}{l}\mathrm{R}^{2}=0.96 ; \mathrm{MAE}=1.4-3.4 \\
\mathrm{~g}-\mathrm{H}_{2} \mathrm{~L}^{-1} ; \mathrm{RMSE}=3.1-4.4 \\
\mathrm{~g}-\mathrm{H}_{2} \mathrm{~L}^{-1}\end{array}$ \\
\hline $\begin{array}{l}\text { Borboudakis } \\
\text { et al. }(2017)^{63}\end{array}$ & $\begin{array}{l}92 \text { binary features based on linker, } \\
\text { metal cluster, and } 12 \text { functional } \\
\text { groups }\end{array}$ & $\begin{array}{l}\text { Ridge linear regression } \\
\text { and support vector ma- } \\
\text { chine with polyno- } \\
\text { mial/Gaussian ker- } \\
\text { nel }^{77,79,80}\end{array}$ & $\begin{array}{l}\text { Total } \mathrm{H}_{2} \text { storage capacity at } 1 \\
\text { bar and } 77 \mathrm{~K}\end{array}$ & $\begin{array}{l}\mathrm{AUE}=0.47 \text { (ridge regres- } \\
\text { sion), } 0.50(\mathrm{SVM}) \mathrm{g}-\mathrm{H}_{2} \mathrm{~g}^{-1}- \\
\mathrm{MOF}\end{array}$ \\
\hline $\begin{array}{l}\text { Thornton et } \\
\text { al. }(2017)^{78}\end{array}$ & $\begin{array}{l}\text { Adsorption energy, } \rho_{\text {crys }}, v f \text {, gsa, vsa, } \\
\text { lcd }\end{array}$ & Neural network ${ }^{72}$ & $\begin{array}{l}\text { Net } \mathrm{H}_{2} \text { capacity for pressure } \\
\text { swing between } 1 \text { and } 100 \text { bar } \\
\text { at } 77 \text { and } 298 \mathrm{~K}\end{array}$ & $\begin{array}{l}\mathrm{R}^{2}=0.88 ; \mathrm{RMSE}=3.6 \mathrm{~g}-\mathrm{H}_{2} \\
\mathrm{~L}^{-1}\end{array}$ \\
\hline
\end{tabular}

The database of MOF properties ${ }^{71}$ generated in these prior studies presents an opportunity to develop ML models that can predict $\mathrm{H}_{2}$ uptake across even larger MOF datasets. ${ }^{1,71}$ Table 1 summarizes prior ML studies of $\mathrm{H}_{2}$ storage in MOFs. (Reports employing ML for other adsorbates such as $\mathrm{CH}_{4},{ }^{72,73} \mathrm{CO}_{2}{ }^{74,75}, \mathrm{~N}_{2}{ }^{74,75}$ are summarized in Table S2.) To the best of our knowledge, ML was first used to predict $\mathrm{H}_{2}$ uptake in compounds from the Nanoporous Materials Genome. ${ }^{76}$ A neural network $(\mathrm{NN})^{77}$ was used to predict usable capacities on a test set of $\sim 1000$ compounds, including MOFs. ${ }^{78}$ In the same year, Borboudakis et al. ${ }^{63}$ predicated $\mathrm{H}_{2}$ capacities in 100 MOFs using 92 binary features related to a MOF's linker, metal cluster, and functional group (s). Ridge linear regression $(\mathrm{RR})^{77,79,80}$ and support vector machine (SVM) $)^{77,81}$ algorithms were used to predict gravimetric capacity. Later, Bucior et $\mathrm{al}^{82}$ predicted the $\mathrm{H}_{2}$ capacities of 50,000 MOFs extracted from the CSD using multi-linear regression (MLR). ${ }^{77}$ The models were trained using the energetics of $\mathrm{H}_{2-}$ MOF interactions and the usable volumetric capacities predicted by GCMC. More recently, ML was used to predict $\mathrm{H}_{2}$ storage capacities in 105 hypothetical MOFs constructed from 17 different topologies, 4 distinct metal clusters, and 5 unique organic linkers. ${ }^{43} \mathrm{NN}^{77}$

models employing 11 features were trained to predict total volumetric uptake at various temperatures and pressures. ${ }^{43}$

Expanding upon these prior reports, the present study applies ML to explore a large database of 918,734 known and proposed MOFs. The database was assembled from a diverse collection of publicly-available MOF repositories, ${ }^{1,29,41-45,83,84,31,33,34,36-40}$ and allows for a wide-ranging and consistent assessment of $\mathrm{H}_{2}$ uptake in MOFs.

Here the extremely randomized trees (ERT) ${ }^{77,85}$ algorithm was identified as the most accurate ML model for predicting $\mathrm{H}_{2}$ uptake. A training set comprising 24,674 MOFs was sufficient to enable accurate predictions of usable capacities across 820,039 unseen compounds. $^{71}$ These predictions were made using a small set of seven crystallographic features as input: single-crystal density, pore volume, gravimetric \& volumetric surface area, void fraction, largest cavity diameter, and pore limiting diameter. Importantly, ML identified 8,282 MOFs - 8,187 appropriate for pressure swing operation and 95 for temperature/pressure swing use - with the potential to exceed both the gravimetric and volumetric capacities of state-of- the-art materials. These compounds are comprised predominantly of hypothetical MOFs, and exhibit low densities $\left(<0.31 \mathrm{~g} \mathrm{~cm}^{-3}\right)$ in combination with high surface areas $\left(>5,300 \mathrm{~m}^{2} \mathrm{~g}^{-1}\right)$, void fractions $(\sim 0.90)$, and pore volumes $\left(>3.3 \mathrm{~cm}^{3} \mathrm{~g}^{-1}\right)$. In addition to identifying high-capacity MOFs, the relative importance of the input features is quantified; dependencies on the ML algorithm and training set size and are also assessed. The single most important features for predicting $\mathrm{H}_{2}$ uptake are pore volume (for gravimetric capacity) and void fraction (for volumetric capacity). A simplified model using only two input features is demonstrated to predict capacities with high accuracy - within 0.2 wt.\% and $1.4 \mathrm{~g} \mathrm{~g}-\mathrm{H}_{2} \mathrm{~L}^{-1}$ of more expensive Monte Carlo calculations. The ML models are available for use via the web, ${ }^{86}$ allowing for rapid and accurate predictions of hydrogen capacities with only a small amount of structural data required as input.

\section{METHODOLOGY}

MOF database. A database of crystal structures for 918,734 MOFs was created by combining 19 existing databases. ${ }^{1,29,41-}$ 45,83,84,31,33,34,36-40 Table 2 summarizes the source databases and the number of MOFs contained in each. Out of these 19 databases, only the $\mathrm{UM}^{31}{ }^{31} \mathrm{CSD},{ }^{29,30}$ and $\mathrm{CoRE}^{33,34}$ databases contain data on MOFs that have been previously synthesized. (MOFs listed in these datasets are referred to as 'real' MOFs.) The remaining databases contain data for proposed, or 'hypothetical,' MOFs. The combined database includes data for seven crystallographic properties that were calculated using the zeo++ code. ${ }^{25,87-90}$ These properties include: single crystal density $(\mathrm{d})$, pore volume $(\mathrm{pv})$, gravimetric surface area (gsa), volumetric surface area (vsa), void fraction (vf), largest cavity diameter (lcd), and pore limiting diameter (pld).

A prior study examined a subset of the present database, wherein the hydrogen uptake in 495,305 MOFs was estimated using the Chahine rule. ${ }^{11,1,2}$ Subsequently, usable uptake in a portion of this subset comprising 43,777 MOFs predicted to be promising based on 
Table 2. MOF datasets employed in this study.

\begin{tabular}{|c|c|}
\hline Source & Number of MOFs \\
\hline $\begin{array}{l}\text { Real MOFs: } \\
\mathrm{UM}^{31}+\mathrm{CoRE}^{33}+\mathrm{CSD}^{29,30}\end{array}$ & 15,235 \\
\hline CoRE $2019^{34}$ & 14,142 \\
\hline${ }^{*}$ CSD 2017 additional ${ }^{29,30}$ & 48,696 \\
\hline Mail-order ${ }^{38}$ & 112 \\
\hline In-silico deliverable ${ }^{46}$ & 2,816 \\
\hline In-silico surface $e^{39}$ & 8,885 \\
\hline MOF-74 analogs ${ }^{40}$ & 61 \\
\hline ToBaCCo ${ }^{59}$ & 13,512 \\
\hline Zr-MOFs ${ }^{45}$ & 204 \\
\hline Northwestern $^{36}$ & 137,000 \\
\hline${ }^{* *}$ Univ. of Ottawa ${ }^{39,91,92}$ & 317,462 \\
\hline $\mathrm{BJT}_{\mathrm{MOFs}}{ }^{83}$ & 303,793 \\
\hline${ }^{* * *}$ R-WLLFHS ${ }^{43,93}$ & 51,163 \\
\hline MTV $^{84}$ & 11,555 \\
\hline CSM-2018-I ${ }^{44}$ & 117 \\
\hline CSM-2018-II ${ }^{45}$ & 32 \\
\hline CSM-2019-I ${ }^{46}$ & 99 \\
\hline In-house ${ }^{1}$ & 18 \\
\hline Total & 918,734 \\
\hline \multicolumn{2}{|c|}{$\begin{array}{l}\text { A subset of the CSD } 2017 \text { MOF dataset }{ }^{29,30} \text { whose crystallographic properties } \\
\text { were found to exhibit extremely low values (e.g GSA } \sim 0) \text { in a prior study. }\end{array}$} \\
\hline
\end{tabular}

the Chahine rule was evaluated using Grand Canonical Monte Carlo (GCMC). This GCMC-evaluated dataset contained a mix of real and hypothetical MOFs: 15,235 real MOFs were sourced from the $\mathrm{UM},{ }^{31} \mathrm{CoRE}^{33}$ and Cambridge Structural Databases, ${ }^{29,30}$ and 28,542 hypothetical MOFs were extracted from the Mail-order, ${ }^{38}$ in silico deliverable, ${ }^{46}$ in silico surface, ${ }^{39} \mathrm{MOF}-74$ analogs, ${ }^{40} \mathrm{ToBaCCo},{ }^{59} \mathrm{Zr}$ MOFs, ${ }^{45}$ Northwestern, ${ }^{36}$ University of Ottawa, ${ }^{37,91,92}$ and in-house ${ }^{1}$ hypothetical MOF databases (see Ref. 1 or Table S1 for details). ${ }^{1,29,31,33,36-40}$ Hydrogen uptake isotherms for two operating conditions were predicted: for an isothermal pressure swing (PS) at $\mathrm{T}=$ $77 \mathrm{~K}$ between 5 and 100 bar, and for a combined temperature-pressure swing (TPS) between $77 \mathrm{~K} / 100 \mathrm{bar}$ (filled state) and $160 \mathrm{~K} / 5$ bar (empty state). Usable gravimetric (UG) and volumetric (UV) capacities were then calculated based on the isotherm data.

In addition to the 43,777 MOFs examined in Ref. 1 , in the present study GCMC isotherms were evaluated for an additional 54,918 MOFs. These MOFs were selected at random from the 495,305 entry HyMARC database and therefore represent a more diverse sampling of the MOF property-space. To this dataset 423,429 additional compounds were added from 7 additional datasets: BJT MOFs, ${ }^{83}$ R-WLLFHS, ${ }^{41,93}$ MTV, ${ }^{84}$ CSM-2018-I, ${ }^{42}$ CSM2018-II, ${ }^{43}$ and CSM-2019-I, ${ }^{44}$ and selected MOFs from the CSD 2017 dataset. ${ }^{29,30}$ Subsequently, the capacities of the MOFs from these additional datasets were predicted by the ML models without retraining (i.e., no MOFs from these datasets were used for training or testing). In total, the dataset employed in the present study contains $\mathrm{H}_{2}$ uptake data for $98,695 \mathrm{MOFs}^{71}$ and crystallographic property data for 918,734 MOFs.

Machine learning model. The No Free Lunch Theorem ${ }^{94}$ implies that the optimal choice of ML algorithm is problem-specific. The differing performance of the algorithms summarized in Tables 1 and $S 2$ is consistent with this notion. Identifying the best algorithm for a given dataset requires comparing multiple $\mathrm{ML}$ methods, each with optimized hyperparameters. Unfortunately, few comparisons
Table 3. Machine learning regression algorithms employed in this work.

\begin{tabular}{|c|c|}
\hline Machine Learning Algorithm & Abbreviation \\
\hline Extremely Randomized Trees ${ }^{77,85104,105}$ & ERT \\
\hline Boosted Decision Trees $77,102,104,105-107$ & BDT \\
\hline Bagging with Decision Trees ${ }^{77,104,105,108}$ & $\mathrm{~B} / \mathrm{DT}$ \\
\hline Random Forest ${ }^{77,104,105,109}$ & $\mathrm{RF}$ \\
\hline Bagging with Random Forest $77,104,105,108,109$ & $\mathrm{~B} / \mathrm{RF}$ \\
\hline Gradient Boosting $77,95,102,104,105,107$ & GB \\
\hline Decision Trees ${ }^{77,104,105}$ & DT \\
\hline
\end{tabular}

$\mathrm{Nu}$-Support Vector Machine with Radial Basis Function (RBF) Kernel $\mathrm{l}^{77,81,96-98,104,105}$

Support Vector Machine Radial Basis Function (RBF)

Kernel ${ }^{77,81,96-98,104,105}$

$\mathrm{Nu}-\mathrm{SVM} / \mathrm{RBF}-\mathrm{K}$

SVM/RBF-K $99,104,105$

Linear Regression $77,79,80,104,105$

SVM/L-K

Ridge Regression $77,79,80,104,105$

K-Nearest Neighbors ${ }^{77,99,101,104,105}$

AdaBoost ${ }^{77,102,104,105-107}$
LR

$\mathrm{RR}$

K-NN

$\mathrm{AB}$ of ML methods for gas adsorption exist; although dozens of ML algorithms are available,,$^{77,79,100-109,80,81,85,95-99}$ only $\mathrm{RR},{ }^{77,79,80} \mathrm{MLR},{ }^{77}$ $\mathrm{SVM}^{77,81}$ and $\mathrm{NN}^{77}$ have been examined for predicting $\mathrm{H}_{2}$ storage. ${ }^{43,63,78,82,108}$ The present study casts a wider net by comparatively assessing $14 \mathrm{ML}$ algorithms (Table 3). ${ }^{77,79,100-109,80,81,85,95-99}$

The crystallographic properties of MOFs are known to correlate with $\mathrm{H}_{2}$ capacities. ${ }^{2,31,110-114}$ The ML models developed here exploit these correlations by adopting only crystallographic properties as input features. Moreover, the number of features was restricted to a small set comprising 7 properties: d, pv, gsa, vsa, vf, lcd, and pld. These are the same properties employed in our prior work. ${ }^{1,2,87,115}$ Figure S1 shows the distribution of crystallographic properties for the training, test, and unseen datasets. Also, Table S3 summarizes five descriptive (minimum, maximum, mean, median, and \% of 0 's) and two distribution statistics (skew and kurtosis) of all crystallographic features for the training, test, and unseen datasets. (The details regarding these statistics and the definitions of skew and kurtosis can be found in Section S3 of the SI). The maxima's and minima's of the features in the training set establish the validity ranges of the ML models developed here.

The goal of the ML models is to predict 4 output properties: UG and UV for each of PS and TPS operating conditions. This was accomplished by developing separate ML models for each of the four targeted capacities. Figure S2 illustrates the overall work-flow.

The existing dataset of 98,695 MOFs (for which both crystallographic and capacity data are available $)^{71}$ was initially split into training and test sets of 74,201 and 24,674 MOFs, respectively, after shuffling the entire dataset. ${ }^{109}$ ML algorithms ${ }^{95,98,99,109}$ (Table 3) were implemented using the scikit-learn library. ${ }^{109}$ Both scaled and unscaled features were used in training ML models. 10-fold cross-validation was used to optimize the hyperparameters of each model. The performance of the ML algorithms was assessed by comparing the predicted $\mathrm{H}_{2}$ capacities to the capacity predicted by GCMC for the MOFs in the test set. The metrics used for the performance assessment of ML models were the coefficient of determination $\left(\mathrm{R}^{2}\right)$, average unsigned error (AUE), root-mean-squared error (RMSE), median absolute error (MAE), mean absolute percentage error (MAPE), and the Kendall rank correlation coefficient $(\tau)$. Additional details regarding these calculations can be found in Section S5 of the SI. 


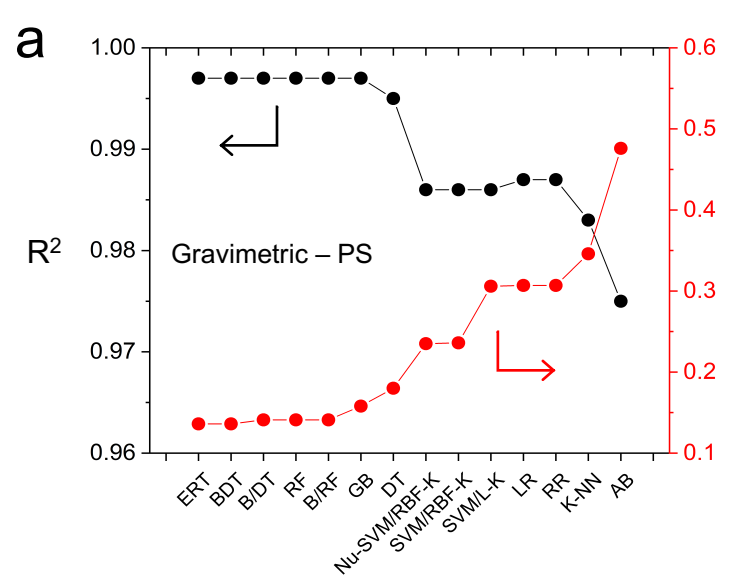

ML algorithm

C

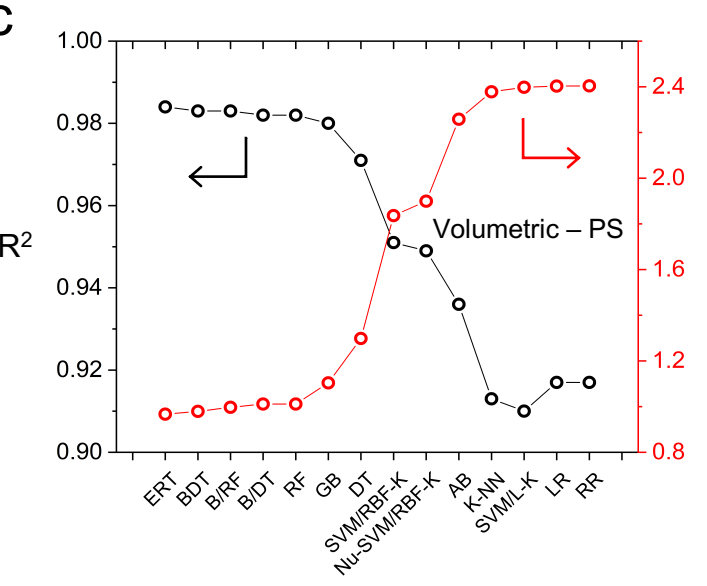

ML algorithm

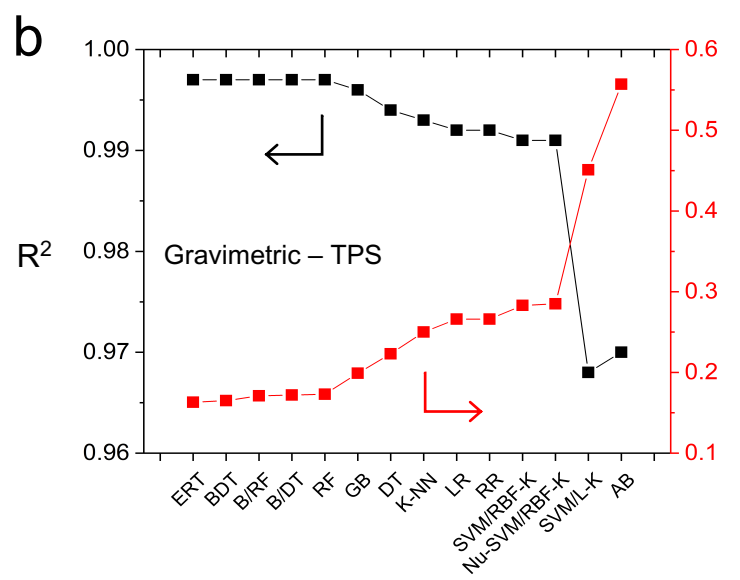

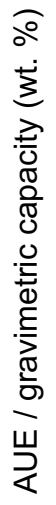

ML algorithm

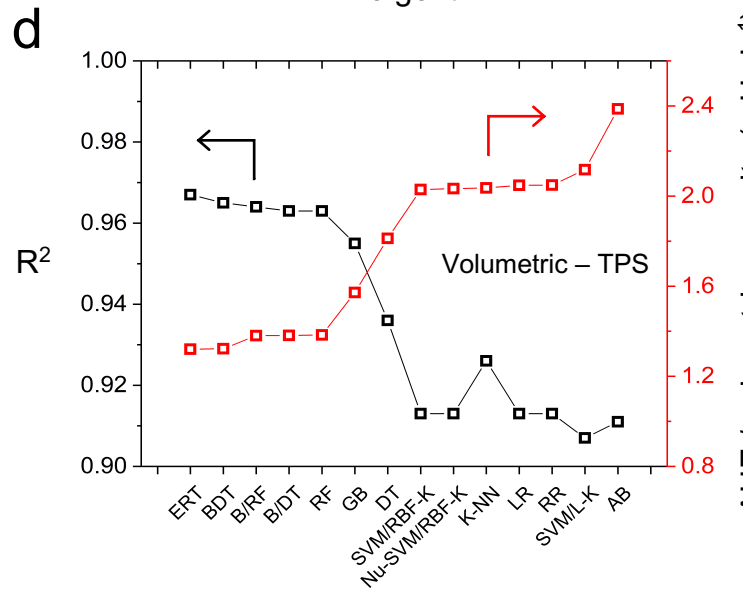

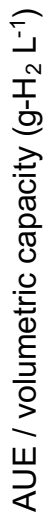

Figure 1. Comparison of $M L$ algorithms for predicting hydrogen uptake in MOFs. Left $(a, c)$ and right $(b, d)$ panels report performance for PS and TPS conditions, respectively. Top $(\mathrm{a}, \mathrm{b})$ and bottom $(\mathrm{c}, \mathrm{d})$ panels report performance for usable gravimetric and volumetric capacities, respectively. The abbreviations for the ML methods are defined in Table 3.

Dataset size. An obstacle to wider adoption of ML in materials science is the availability of sufficient quantities of high-quality training data. ${ }^{116,117}$ Unfortunately, it is not yet clear how much data is needed to construct a useful ML model for a given system. Fernandez et al. ${ }^{73}$ found that a reasonable balance between accuracy $\left(\mathrm{R}^{2}\right.$ $\sim 0.85$ to 0.93 ) and computational expense for predicting methane storage in MOFs was achieved for a training set containing data on 10,000 MOFs with 3 features. In contrast, Fanourgakis et al. ${ }^{118}$ showed that a much smaller training set of $\sim 1000$ MOFs was sufficient to predict methane uptake when using six crystallographic fea- tures and four fictitious features. The different training set sizes required in these prior studies arise from the differing numbers and types of features used.

The present study explores this issue further by systematically examining the effect of training set size, and the training set to test set ratio, on ML accuracy. For each of the four targeted capacity outputs, 100 independent $\mathrm{ML}$ models were developed by varying the size of the training set between 100 and 74,000 MOFs (see Table S4 for a list of the training set sizes). The four best-performing ERT ML algorithms identified earlier were used with 10 -fold cross-validation.

Table 4. Performance of the Extremely Randomized Trees ML algorithm in predicting UG and UV $\mathrm{H}_{2}$ capacities of MOFs under PS and TPS conditions. $R^{2}$, AUE, RSME, and MAE represent the coefficient of determination, average unsigned error, root-meansquared error, and median absolute error, respectively.

\begin{tabular}{cccccc}
$\mathbf{H}_{2}$ capacity type & $\mathbf{R}^{2}$ & $\begin{array}{c}\text { AUE } \\
\text { (capacity units) }\end{array}$ & $\begin{array}{c}\text { RMSE } \\
\text { (capacity units) }\end{array}$ & Kendall $\tau$ & $\begin{array}{c}\text { MAE } \\
\text { (capacity units) }\end{array}$ \\
UG at PS $($ wt. \%) & 0.997 & 0.14 & 0.18 & 0.961 & 0.10 \\
UV at PS $\left(\mathrm{g}-\mathrm{H}_{2} \mathrm{~L}^{-1}\right)$ & 0.984 & 0.97 & 1.40 & 0.922 & 0.69 \\
UG at TPS $($ wt. \%) & 0.997 & 0.16 & 0.23 & 0.966 & 0.10 \\
UV at TPS $\left(\mathrm{g}-\mathrm{H}_{2} \mathrm{~L}^{-1}\right)$ & 0.967 & 1.32 & 1.92 & 0.819 & 0.91 \\
\hline
\end{tabular}



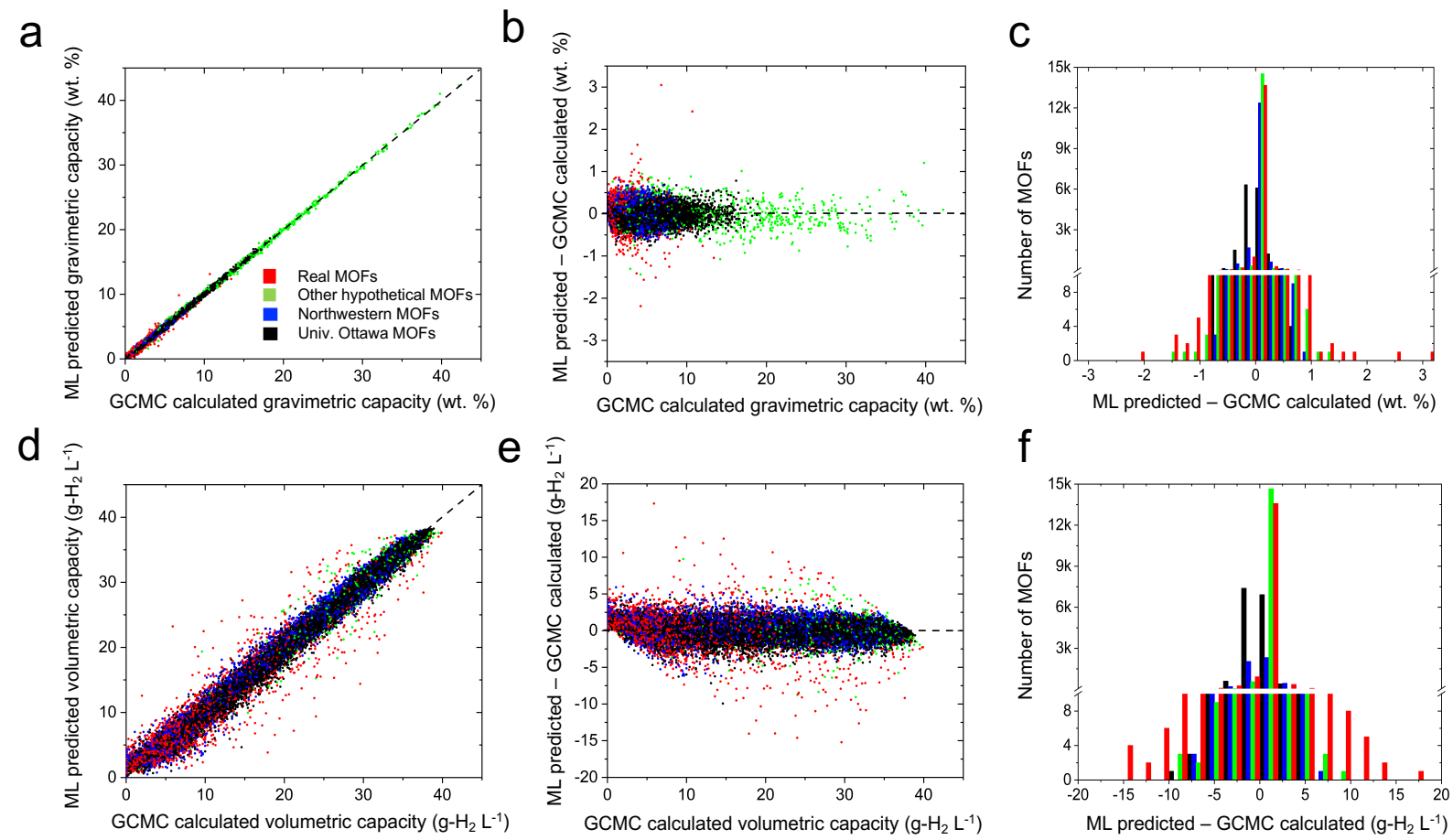

Figure 2. Performance of the Extremely Randomized Trees ML algorithm with respect to GCMC calculations for predicting usable $\mathrm{H}_{2}$ capacities in MOFs. Data is collected at $77 \mathrm{~K}$ for a pressure swing between 100 and 5 bar on a test set of 24,674 MOFs. Different colors represent different categories of MOFs. Top (a-c) and bottom (d-f) panels illustrate performance for usable gravimetric and volumetric capacities, respectively. (a, d): Agreement between ML and GCMC predictions. (b, e): Difference between ML and GCMC as a function of GCMC capacity. (c, f) Distribution of differences in predictions between ML and GCMC.

The resulting models were assessed using a common test set of 24,674 MOFs.

Feature importance/selection. The well-known Chahine rule proposes a linear correlation between gravimetric surface area and excess gravimetric $\mathrm{H}_{2}$ capacity in adsorbents. ${ }^{119,120}$ Nevertheless, the Chahine rule overpredicts $\mathrm{H}_{2}$ capacities for MOFs with high surface areas, ${ }^{120}$ and has not been extended to predict usable capacities. ${ }^{1,2,6}$ Hence, a model for predicting $\mathrm{H}_{2}$ uptake that is more general than the Chahine rule, yet requires limited input data, would be very helpful. In principle, ML could be used to generate such a predictive model if the features that are the most important for predicting $\mathrm{H}_{2}$ uptake could be identified. Along these lines, Pardakhti et al. reported improved accuracy in predicting $\mathrm{CH}_{4}$ adsorption when using a combination of (7) crystallographic and (19) chemical features. ${ }^{72}$ Recently, Moosavi et al. explored feature importance in predicting the synthesis of MOFs. ${ }^{121}$

The present study determines the minimum number and optimal combination of crystallographic features necessary to achieve a specified accuracy in predicting $\mathrm{H}_{2}$ uptake. The relative importance of the input features was assessed for all possible univariate and multivariate feature combinations using ERT ML models. The number of multivariate feature combinations, $M$, is given by: $M\left(n_{\text {tot }}, n_{\text {sub }}\right)=\frac{n_{\text {tot }} !}{n_{\text {sub }} !\left(n_{\text {tot }}-n_{\text {sub }}\right) !}$, where $n_{\text {tot }}=7$ is the total number of available features, and $1<=n_{s u b}<=7$ is the number of features used as input to a given ML model. A total of 127 feature combinations are possible. ML models were developed for each of these feature combinations for each of the 4 output capacities, result- ing in a total of 508 distinct ML models. All models were trained using a dataset of 74,021 MOFs and tested on a common set of 24,674 MOFs. 10-fold cross-validation was used for tuning and validating the models using only the training set. Univariate feature importance was further assessed using (i.) Pearson's correlation coefficient (r), ${ }^{122-124}(i i)$, Breiman and Friedman's tree-based algorithm as implemented in Scikit-learn, ${ }^{95,109}$ and (iii) the permutation importance method as implemented in rfpimp package. ${ }^{125}$ Additional details regarding these methods can be found in Section 11 of the SI.

\section{RESULTS AND DISCUSSION}

Evaluating ML algorithms. Tables S5-S8 illustrate the effect of several feature scaling methods on the performance of the ML algorithms examined here. Only the SVM family of models (SVM/L-K, SVM/RBF-K, and Nu-SVM/RBF-K) $)^{77,95,101,103,104,109}$ were impacted by the choice of scaling method.

Figure 1 compares the accuracy of the ML algorithms for predicting hydrogen uptake in MOFs. $\mathrm{R}^{2}$ and AUE were used as performance metrics. SVM variants were trained using min-max feature scaling; un-scaled features were used in training the remaining models. The performance of the algorithms as measured by 4 additional metrics - root mean square error (RMSE), explained variance (EV), MAE (median absolute error), and Kendall $\tau$ - is reported in Tables S5-8.

Overall, these data indicate that the tree-based ensemble methods are superior to the other methods examined. In particular, the Extremely Randomized Trees (ERT) ${ }^{77,85,109}$ algorithm exhibited the best performance overall. Boosted Decision Trees (BDT), ${ }^{77,95-}$ 
Ratio of training to test set

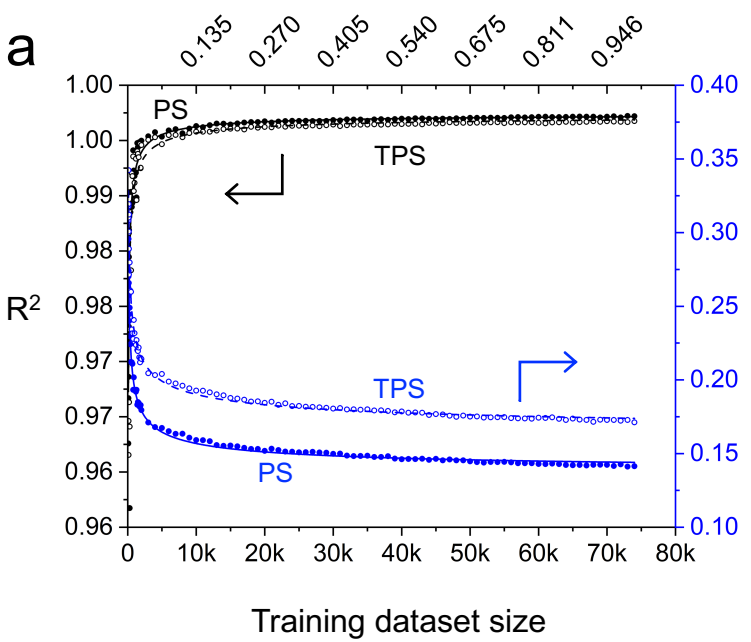

Ratio of training to test set

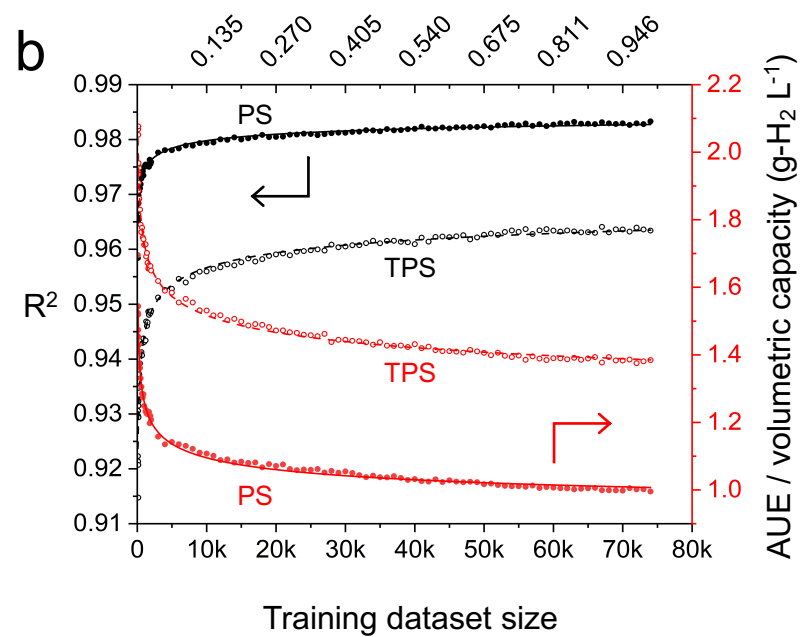

Figure 3. Performance of Extremely Randomized Trees ML models for predicting usable (a) gravimetric and (b) volumetric $\mathrm{H}_{2}$ capacity as a function of training set size and the ratio of training to test set size. 100 different training sets ranging in size between 100 and 74,021 MOFs were examined. A common set of 24,674 MOFs was used for testing. Performance is quantified using $\mathrm{R}^{2}$ (left axis, black) and the average unsigned error, AUE (right axis, blue and red for UG and UV, respectively). Lines represent a power-law fit to the data.

97,107,109 Random Forest (RF), ${ }^{77,99,109}$ and Bagging algorithm variants $^{77,98,109,126,127}$ (with tree-based base estimators) are nearly as accurate. The $\mathrm{R}$ values for ERT predictions exceed 0.997 for gravimetric capacities, which are equivalent to errors of $\sim 0.14$ wt.\%. Volumetrically, the accuracy of the ERT algorithm is slightly worse than its gravimetric performance: $\mathrm{R}^{2}=0.967-0.984$, equivalent to errors of $\sim 1.1 \mathrm{~g}-\mathrm{H}_{2} \mathrm{~L}^{-1}$ on average. In general, the worst-performing algorithms were linear regression, ridge regression, and support vector machine with linear kernel. For these algorithms $\mathrm{R}^{2}$ varies between 0.913 and 0.992 depending on the conditions (i.e., gravimetric/volumetric and PS/TPS). As expected, the linear nature of these algorithms fails to fully capture the nonlinear dependence of output capacities on the multiple input features.

Figure 1 also shows that all the algorithms tested yield more accurate predictions of UG capacities compared to those for UV. Likewise, all algorithms more accurately predict usable capacities under PS conditions than under TPS conditions. This reflects the fact that the functional relationships between output capacities (UG/UV) and input features under PS and TPS conditions are likely different, as was observed in previously reported structure(feature)-property(capacity) relationships. ${ }^{1,6,128}$

Table 4 summarizes the performance of the ERT algorithm in further detail. A comparison of Tables 1 and 4 indicates that the accuracy of the present ML models surpass previously-reported models for $\mathrm{H}_{2}$ uptake. Furthermore, the present models also appear to be an improvement over earlier models that aim to predict the adsorption capacities of MOFs for any gas species, Table S2. This improved performance can be attributed to the exploration and optimization of multiple ML algorithms, use of an appropriate feature set, and the relatively large size of the present training set.

Figure 2 illustrates the degree of agreement between ERT ML predictions and GCMC calculations of usable $\mathrm{H}_{2}$ capacities under PS conditions as a function of MOF source database. (Fig. S3 shows similar data for TPS conditions; see also Table 4.) As mentioned above, the present ML models more accurately predict UG capacities than UV capacities. The largest differences between ML and GCMC capacities (Fig. 2c,f \& Fig. S3c,f) primarily occur for the real MOF dataset. In principle, these differences may arise either from ML overfitting or from inaccurate GCMC predictions caused by non-ideal/incomplete MOF crystal structure data (i.e., missing atoms, disorder, etc.) as mentioned in prior studies. ${ }^{1,35,129-132}$ ERT algorithms are fairly robust against overfitting. ${ }^{85}$ To examine the possibility for overfitting, test set errors were compared with training set errors as shown in Fig. S4 and Table 4. These data suggest that the outliers are not a consequence of over fitting; hence, inaccuracies in the crystal structure data is proposed as the most likely source of this disagreement. ${ }^{1,35,129-132}$

Effect of training set size. Figure 3 illustrates the impact of training set size on the accuracy of the ERT ML models as quantified using $\mathrm{R}^{2}$ and AUE. (Table S4 summarizes the dataset sizes used in these plots.) For training sets containing more than 5000 MOFs $\mathrm{R}^{2}$ and AUE vary slowly and in a monotonic fashion, with AUE decreasing and $\mathrm{R}^{2}$ increasing. The accuracy of the models is more sensitive to the size of the training set for smaller training sets containing roughly 5,000 or fewer MOFs. Figure S5 highlights the variation in performance for these smaller training sets.

The trends AUE as a function of training set size can be fit to a power-law expression of the form $\operatorname{AUE}(\mathrm{m})=\alpha \mathrm{m}^{\beta}+\gamma$, where $\mathrm{m}$ represents the size of the training set and $\beta$ is the power law exponent. Fitting this model to the data shown in Fig. 3 reveals that the AUE for UG converges faster with training set size $(\beta=-0.37$ and -0.43$)$ than it does for UV $(\beta=-0.16$ and -0.23$)$. A full tabulation of the power-law parameters is given in Table S9. Based on these powerlaw expressions, one can determine the necessary size of the training set to achieve a desired level of accuracy. For example, assuming pressure swing operation, to achieve an AUE of approximately 0.25 wt.\% and $1.5 \mathrm{~g}-\mathrm{H}_{2} \mathrm{~L}^{-1}$ requires training set sizes (for UG and UV) of less than 300 MOFs randomly selected from the diverse datasets used here. 

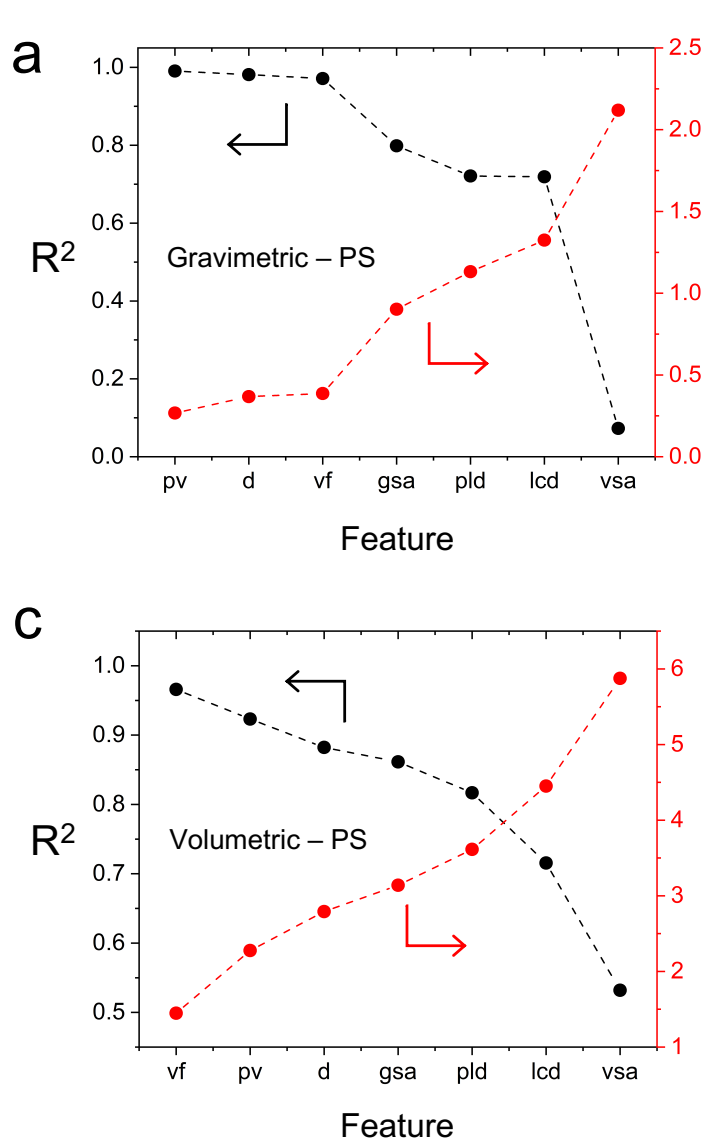
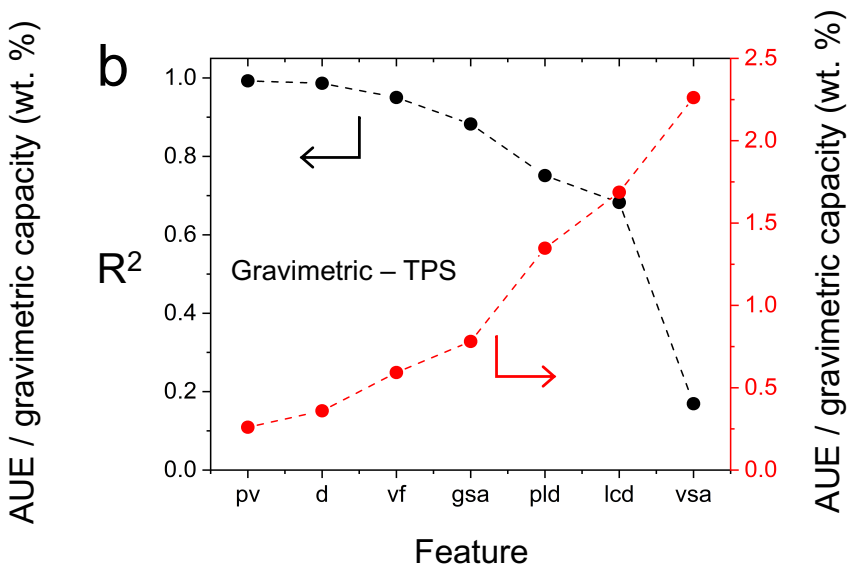

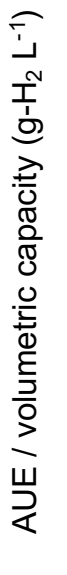

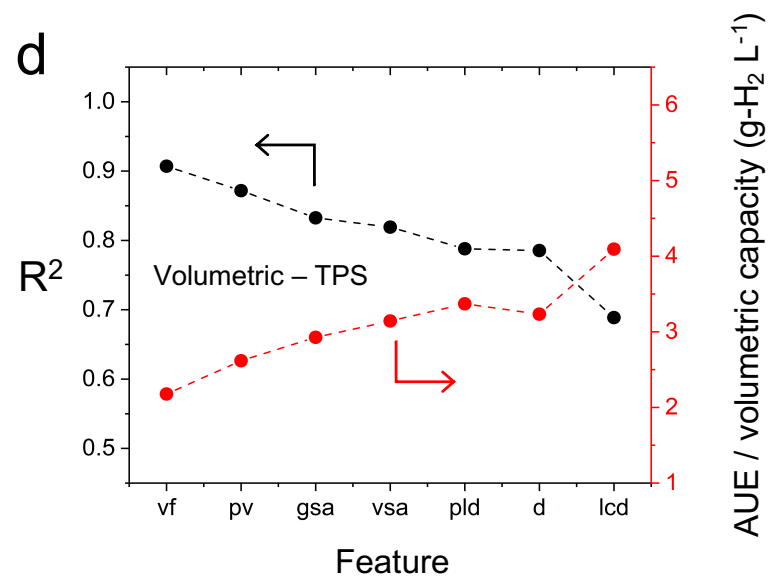

Figure 4. Importance of seven features in predicting usable $\mathrm{H}_{2}$ storage capacities of MOFs. Feature importance was determined by developing distinct ERT models for each individual feature. The accuracy of the resulting models was assessed using $\mathrm{R}^{2}$ (left axis; black data set) and AUE (right axis; red data set). Models were trained on a dataset of 74,201 MOFs and tested on a set of 24,674 MOFs. pv = pore volume; $d=d e n s i t y ; ~ v f$ = void fraction; gsa = gravimetric surface area; pld = pore limiting diameter; lcd = limiting cavity diameter; vsa = volumetric surface area.

Univariate feature importance. Figure 4 illustrates the relative importance of the seven crystallographic features in predicting usable hydrogen uptake in MOFs. Feature importance was determined by developing ERT models for each single feature individually. Additional details for these models are provided in the Supporting Information. Based on these models, it is evident that pore volume (pv) and void fraction (vf) are the dominant features in predicting $\mathrm{H}_{2}$ capacity; these two properties appear as the first- or second-most important single features regardless of operating condition or capacity type. The importance of these features can be rationalized by two factors. First, based on the empirical Chahine rule, the pore volume of a MOF correlates with its excess uptake. ${ }^{133}$ Second, pore volume and void fraction are related $\left(\right.$ since $\left.\mathrm{pv}=\mathrm{vf} \mathrm{d}^{-1}\right)-$ MOFs with larger pv have larger vf, and vice versa. ${ }^{1}$

Conversely, the largest cavity diameter (lcd) and volumetric surface area (vsa) are the single features whose ML models yield the lowest accuracy. The relative importance of the individual features for predicting UG capacities is: $\mathrm{pv}>\mathrm{d}>\mathrm{vf}>$ gsa $>$ pld $>$ lcd $>$ vsa. This ordering is the same for PS and TPS conditions. In contrast, the importance ordering for UV capacities differs based on the operating condition. Nevertheless, vf and pv remain the two most important single features for both UV conditions, in that order (Fig. 4).
Despite their limited input, the single-feature ML models illustrated in Fig. 4 achieve high accuracy. For example, any of the three independent models for UG-PS based only on pv, d, or vf can predict capacities with $\mathrm{R}^{2}>0.95$ and with AUE of less than $0.5 \mathrm{wt} . \%$. The accuracy and simplicity of the univariate ML models suggest that they can be used to quickly screen new MOFs for their utility in hydrogen storage. To that end, optimized single-feature ML models for the four categories of usable capacities considered here have been made available for use on the web. ${ }^{86}$ These models take as input either pv (for UG predictions) or vf (for UV) of a given MOF. These input data can be quickly calculated from a MOF's crystal structure using modern structure analysis codes. ${ }^{25,87-90,134}$ As shown in Fig. 4, these models can predict UG with an average error of less than 0.4 wt.\%, and UV with errors less than $2.2 \mathrm{~g}-\mathrm{H}_{2} \mathrm{~L}^{-1}$.

Figure $\mathrm{S} 6$ compares the single feature importance assessments based on ERT ML models (as reported in Fig. 4) with three popular methods for determining feature importance: Pearson's correlation coefficient $(r),{ }^{122-124}$ Breiman and Friedman's tree-based algorithm as implemented in Scikit-learn, ${ }^{95,109}$ and the permutation importance method as implemented in rfpimp package. ${ }^{125}$ It is clear that the feature importance methods do not reproduce in detail the rank-ordering of feature importance that is suggested by our ERT ML models. Nevertheless, good agreement is evident more broadly. For example, 
in the case of UG (Fig. S6a,c), the three feature importance methods suggest that in aggregate $\mathrm{pv}$ is the most important feature, while vsa is the least, in agreement with the ERT models (Fig. 4a,b). Similarly, for UV, the importance methods suggest that vf and lcd are among the most and least important features, respectively. This is the same trend found in the univariate ERT models (Fig. 4c,d).

Multivariate feature importance. Figure 5 illustrates how the accuracy of the ML models varies with the number and combination of features. Assuming 7 features, $2^{7}-1=127$ possible combinations exist. For a given number of features, Fig. 5 plots the combination of features resulting in the highest accuracy model. (The supplementary file ("Feature_Combination_ML_Models.xlsx") summarizes the performance for all 508 possible feature combinations and capacity/operating condition types .) As expected, Fig. 5 shows that ML accuracy generally increases as the number of input features increases. As previously discussed, when limited to a single feature, vf yields the best accuracy for predicting UV, while pv is the best choice for UG. When the feature set is extended to 2 features, the combination of $\mathrm{d}$ and $\mathrm{pv}$ is the optimal choice among the $\left(\begin{array}{l}7 \\ 2\end{array}\right)=21$ possible pairs regardless of the capacity (UG vs UV) or operating condition
(PS vs TPS). For larger numbers of features, the optimal feature combination depends upon the operating condition and the capacity type. Based on the AUE, whose value tends to plateau as more features are added, highly accurate ML models can be generated using only 5 input features (Table 5). These data lend further support to the notion that the accuracy of a given ML model depends on both the number and identity of the input features. As a slightly more accurate alternative to the univariate web models described above, a subset of the present multivariate ML models that use 4, 5, and 7 input features are also available on the web. ${ }^{86}$

$\mathrm{H}_{2}$ uptake in unseen MOFs. Figure 6 illustrates the $\mathrm{H}_{2}$ storage capacities of 820,039 MOFs as predicted by the 7 -feature ERT ML models developed here. (This dataset is publicly accessible via HyMARC data hub. ${ }^{71}$ ) These MOFs are referred to as 'unseen,' in that they have not been included in the training or test sets used to develop the models. Figures $6 \mathrm{a}, \mathrm{b}$ show UV capacities as functions of UG capacities under PS and TPS conditions, respectively. Both plots exhibit a rapid increase in UV at low values of UG, and reach a maximum in UV at UG values of approximately $9 \mathrm{wt} . \%$. Beyond the maximum, UV decreases relatively slowly with increasing UG. These
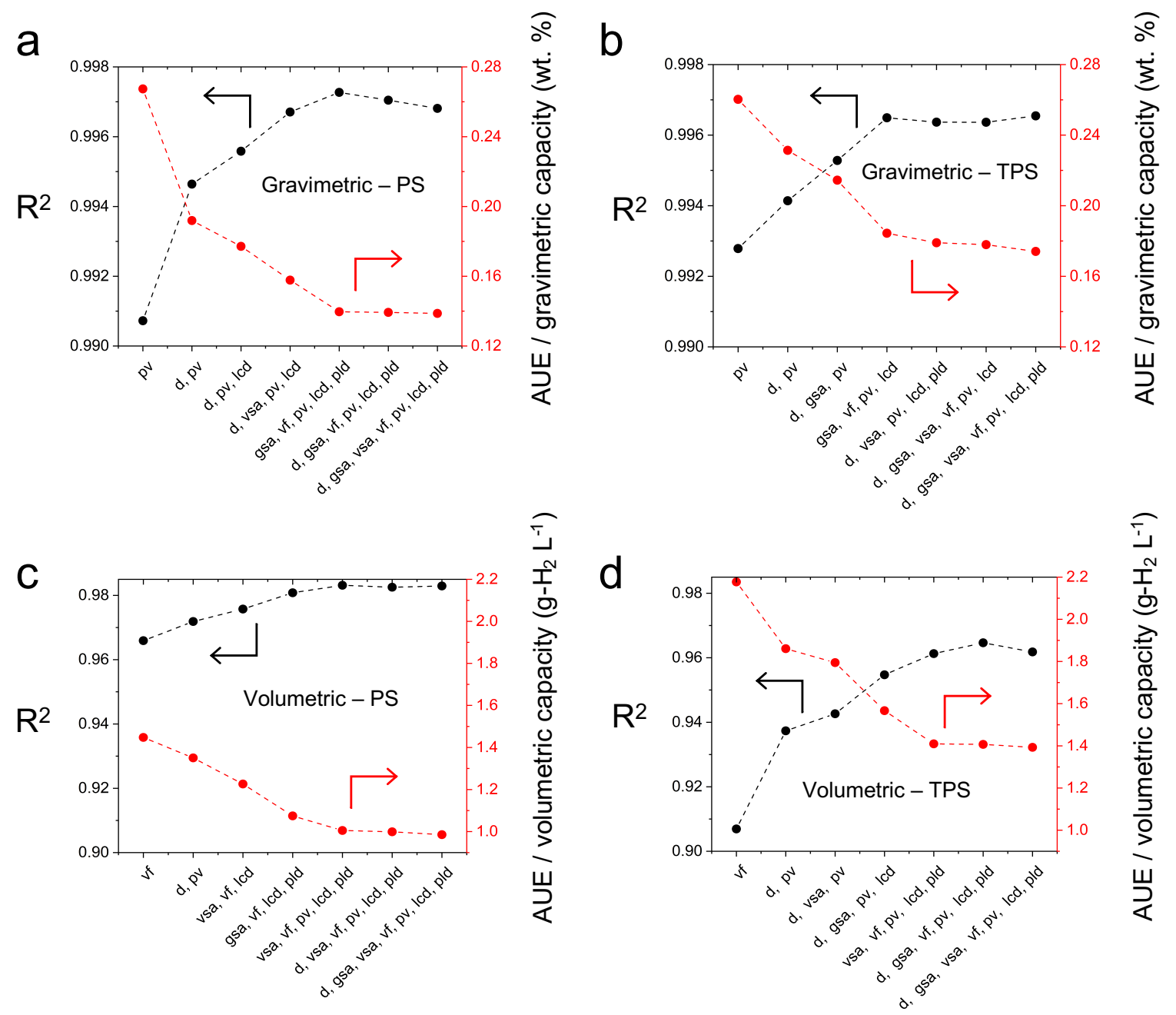

Figure 5. Accuracy of Extremely Randomized Trees (ERT) ML models as determined by $R^{2}$ and AUE as a function of the number and combination of input features. Each data point represents the most accurate feature combination for a given number of features. ERT models were trained on a dataset of 74,201 MOFs. $\mathrm{R}^{2}$ and AUE were calculated using a test of 24,674 MOFs. Feature abbreviations are defined in Fig. 4. 
Table 5. The best combinations of features for predicting UG and UV $\mathrm{H}_{2}$ storage capacities at PS and TPS conditions.

\begin{tabular}{lcccccr} 
Condition & Feature Combination & No. features & $\mathrm{R}^{2}$ & AUE & RMSE & Kendall $\tau$ \\
UG at PS & gsa, vf, pv, lcd, pld & 5 & 0.997 & 0.14 wt. \% & 0.19 wt. \% & 0.959 \\
UG at TPS & d, vsa, pv, lcd, pld & 5 & 0.996 & 0.18 wt. \% & 0.25 wt. \% & 0.959 \\
UV at PS & vsa, vf, pv, lcd, pld & 5 & 0.983 & $1.01 \mathrm{~g}-\mathrm{H}_{2} \mathrm{~L}^{-1}$ & $1.45{\mathrm{~g}-\mathrm{H}_{2} \mathrm{~L}^{-1}}^{-1}$ & 0.920 \\
UV at TPS & vsa, vf, pv, lcd, pld & 5 & 0.961 & $1.41 \mathrm{~g}-\mathrm{H}_{2} \mathrm{~L}^{-1}$ & $2.10 \mathrm{~g}-\mathrm{H}_{2} \mathrm{~L}^{-1}$ & 0.814 \\
\hline
\end{tabular}

trends are consistent with our earlier findings derived from GCMC calculations on smaller datasets. ${ }^{1,2,6}$

In the case of PS operation, the maximum UV across the MOFs in the dataset is $37.4 \mathrm{~g}-\mathrm{H}_{2} \mathrm{~L}^{-1}$; for TPS operation the maximum UV is $48.5 \mathrm{~g}-\mathrm{H}_{2} \mathrm{~L}^{-1}$. In the case of $\mathrm{UG}$, the maximum value predicted is 39 wt.\% for PS operation and 42 wt.\% for TPS. These values can be placed in context by comparing against the DOE hydrogen storage targets, which stipulate system-level hydrogen densities of $5.5 \mathrm{wt} \%$ and $40 \mathrm{~g}-\mathrm{H}_{2} \mathrm{~L}^{-1}$ by 2025 and 6.5 wt. $\% / 50 \mathrm{~g}-\mathrm{H}_{2} \mathrm{~L}^{-1}$ longer-term ('Ultimate target'). ${ }^{6}$ Given that the tank and balance-of-plant for the storage system have non-zero mass and volume, the MOFs examined here cannot meet the Ultimate target for UV, regardless of operating condition. ${ }^{12}$ More optimism exists, however, for meeting the gravimetric targets given the high UG exhibited by these systems on

a

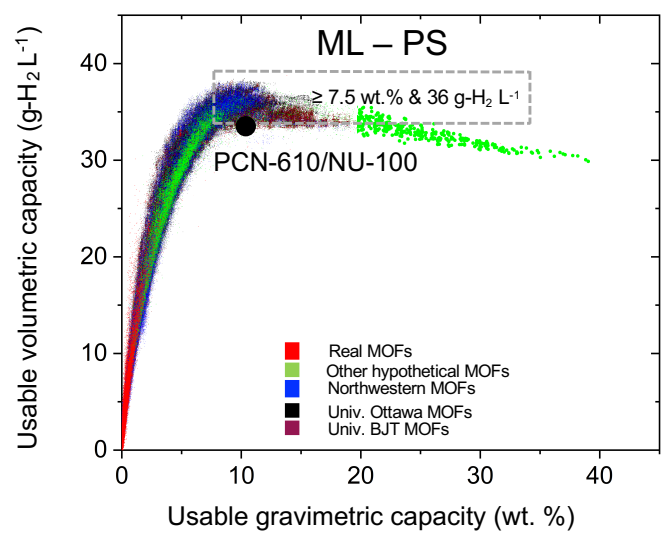

C

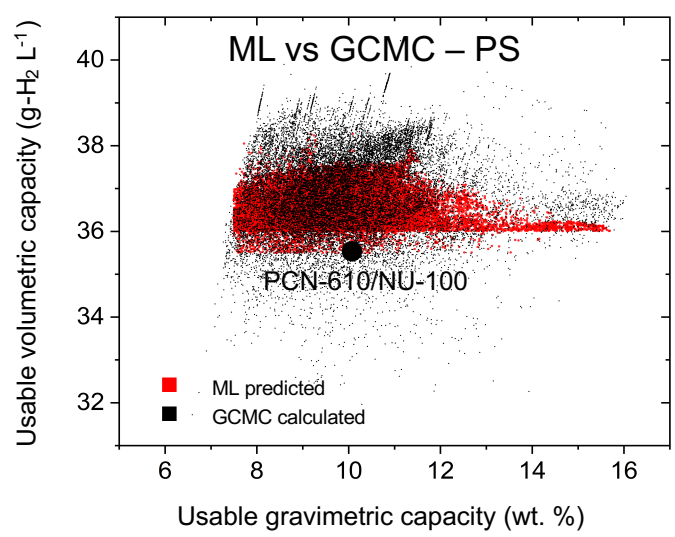

a MOF-only basis. Of course, an additional challenge is to identify MOFs that excel both gravimetrically and volumetrically. ${ }^{1,2,6,31,135}$

It is also helpful to compare the performance predictions in Figs. $6 \mathrm{a}, \mathrm{b}$ with that of state-of-the-art materials. In the case of PS operation, our prior study demonstrated that PCN-610 (NU-100) exhibits a hydrogen capacity of $10.1 \mathrm{wt} . \%$ \& $35.5 \mathrm{~g}-\mathrm{H}_{2} \mathrm{~L}^{-1}, 1$ which to our knowledge is the best combination of gravimetric and volumetric capacities reported for any MOF under these conditions. The data in Figure 6a reveals that 16,345 MOFs can, in principle, exceed this capacity on both a UG and UV basis. In the case of TPS operation (Figure $6 \mathrm{~b})$, MOF-5 remains the benchmark, which a measured capacity of $7.8 \mathrm{wt} . \%$ \& $51.9 \mathrm{~g}-\mathrm{H}_{2} \mathrm{~L}^{-1} .{ }^{2}$ Figure $8 \mathrm{c}$ shows that only 21 MOFs outperform MOF-5 under these conditions.

Regarding the accuracy of the present ML predictions, Table 4 shows that the AUE of these models are on the order of $0.15 \mathrm{wt} . \%$

b
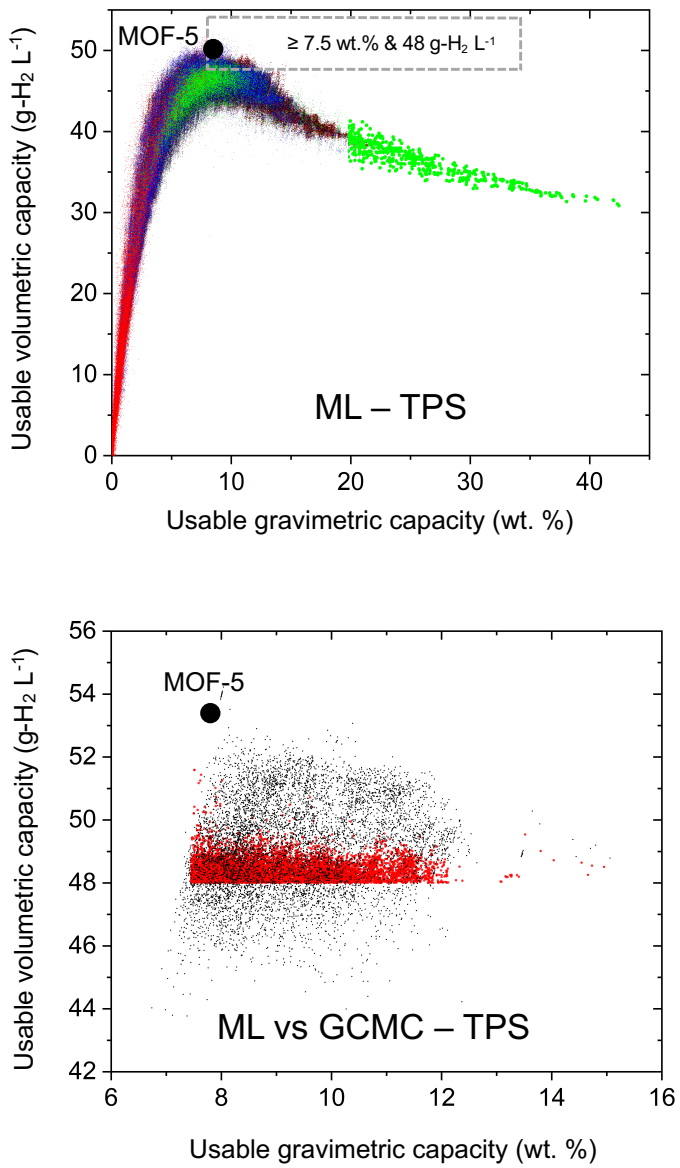

Figure 6. (a,b) Machine learning predictions of usable hydrogen capacities of 820,093 MOFs. Colors indicate the originating database for a given MOF. (c,d) Validation of ML-predicted capacities for the highest capacity MOFs identified by ML (shown in the rectangular regions in c,d) using GCMC simulations. For comparison, the capacities of PCN-610/NU-100 (PS: 10.1 wt.\%, 35.5 g- $\mathrm{H}_{2} \mathrm{~L}^{-1}$ ) and MOF-5 (TPS: 7.8 wt.\%, $51.9 \mathrm{~g}-\mathrm{H}_{2} \mathrm{~L}$ 1) are shown. 
Table 6. Highest capacity MOFs, as identified by ML and verified with GCMC, under pressure swing and temperature + pressure swing conditions. Here NW and UO refer to the Northwestern ${ }^{36}$ and University of Ottawa databases. ${ }^{39}$

\begin{tabular}{|c|c|c|c|c|c|c|c|c|c|c|c|c|}
\hline \multirow{2}{*}{ Name } & \multirow{2}{*}{ Source } & \multirow{2}{*}{$\begin{array}{l}\text { Density } \\
\left(\mathrm{g} \mathrm{cm}^{-3}\right)\end{array}$} & \multirow{2}{*}{$\begin{array}{c}\text { Grav. } \\
\text { surface area } \\
\left(\mathbf{m}^{2} \mathbf{g}^{-1}\right)\end{array}$} & \multirow{2}{*}{$\begin{array}{c}\text { Vol. } \\
\text { surface area } \\
\left(\mathbf{m}^{2} \mathrm{~cm}^{-3}\right)\end{array}$} & \multirow{2}{*}{$\begin{array}{l}\text { Void } \\
\text { fraction }\end{array}$} & \multirow{2}{*}{$\begin{array}{c}\begin{array}{c}\text { Pore } \\
\text { volume }\end{array} \\
\left(\mathrm{cm}^{3} \mathrm{~g}^{-1}\right)\end{array}$} & \multirow{2}{*}{$\begin{array}{c}\text { Largest } \\
\text { cavity } \\
\text { diameter } \\
(\AA)\end{array}$} & \multirow{2}{*}{$\begin{array}{c}\text { Pore } \\
\text { limiting } \\
\text { diameter } \\
(\AA)\end{array}$} & \multicolumn{2}{|c|}{$\begin{array}{l}\text { Usable grav. } \\
\text { capacity (wt. \%) }\end{array}$} & \multicolumn{2}{|c|}{$\begin{array}{c}\text { Usable vol. } \\
\text { capacity }\left(\mathrm{g}-\mathrm{H}_{2} \mathrm{~L}^{-1}\right)\end{array}$} \\
\hline & & & & & & & & & GCMC & ML & GCMC & ML \\
\hline \multicolumn{13}{|c|}{ Pressure swing } \\
\hline mof_7642 & ToBaCCo & 0.30 & 5561 & 1695 & 0.89 & 2.93 & 12.8 & 11.8 & 11.1 & 10.3 & 40.5 & 37.4 \\
\hline mof_7690 & ToBaCCo & 0.30 & 5715 & 1706 & 0.89 & 2.98 & 12.8 & 12.0 & 11.3 & 10.4 & 40.3 & 37.3 \\
\hline mof_7594 & ToBaCCo & 0.40 & 5070 & 2031 & 0.86 & 2.15 & 11.2 & 9.7 & 8.6 & 7.9 & 39.9 & 37.0 \\
\hline mof_7210 & ToBaCCo & 0.29 & 5936 & 1730 & 0.89 & 3.04 & 13.4 & 11.7 & 11.4 & 10.5 & 39.8 & 37.1 \\
\hline mof_7738 & ToBaCCo & 0.25 & 6054 & 1502 & 0.90 & 3.64 & 14.5 & 13.5 & 13.0 & 12.0 & 39.7 & 37.0 \\
\hline hypotheticalMOF_5045702_i_1_j_24_k_20_m_2 & NW & 0.31 & 5926 & 1820 & 0.88 & 2.87 & 16.0 & 11.0 & 10.9 & 10.1 & 39.7 & 37.2 \\
\hline str_m3_o19_o19_f0_nbo.sym.1.out & UO & 0.31 & 5073 & 1583 & 0.90 & 2.88 & 17.7 & 12.9 & 10.8 & 10.1 & 39.7 & 37.1 \\
\hline hypotheticalMOF_5037315_i_1_j_20_k_12_m_1 & NW & 0.31 & 5818 & 1787 & 0.88 & 2.86 & 16.0 & 11.0 & 10.9 & 10.0 & 39.7 & 37.0 \\
\hline hypotheticalMOF_5037467_i_1_j_20_k_12_m_8 & NW & 0.31 & 5860 & 1800 & 0.88 & 2.85 & 16.0 & 11.0 & 10.9 & 10.0 & 39.7 & 37.0 \\
\hline str_m3_o5_o20_fo_nbo.sym.1.out & UO & 0.39 & 4772 & 1882 & 0.87 & 2.22 & 14.1 & 9.6 & 8.7 & 8.1 & 39.7 & 37.2 \\
\hline \multicolumn{13}{|c|}{ Temperature + pressure swing } \\
\hline 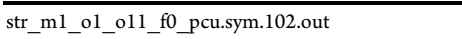 & UO & 0.45 & 4352 & 1974 & 0.84 & 1.84 & 12.9 & 10.1 & 10.4 & 9.7 & 53.1 & 48.1 \\
\hline str_m1_o1_o11_f0_pcu.sym.117.out & UO & 0.47 & 4162 & 1977 & 0.83 & 1.74 & 12.8 & 9.9 & 9.9 & 9.0 & 52.8 & 48.0 \\
\hline str_m1_o1_ol1_fo_pcu.sym.121.out & UO & 0.47 & 4263 & 2006 & 0.83 & 1.76 & 12.1 & 10.2 & 10.0 & 9.4 & 52.7 & 48.1 \\
\hline str_m1_o1_o11_f0_pcu.sym.13.out & UO & 0.46 & 4326 & 2005 & 0.83 & 1.79 & 12.7 & 9.9 & 10.1 & 9.3 & 52.6 & 48.0 \\
\hline str_m1_o1_ol1_f0_pcu.sym.159.out & UO & 0.58 & 3703 & 2138 & 0.80 & 1.38 & 10.4 & 8.6 & 8.3 & 7.6 & 52.6 & 48.5 \\
\hline str_m1_o1_ol1_f0_pcu.sym.200.out & UO & 0.45 & 4359 & 1978 & 0.84 & 1.84 & 12.9 & 10.1 & 10.3 & 9.6 & 52.6 & 48.1 \\
\hline str_m1_o1_ol1_f0_pcu.sym.212.out & UO & 0.60 & 3417 & 2035 & 0.83 & 1.39 & 12.0 & 10.1 & 8.1 & 7.5 & 52.5 & 48.1 \\
\hline str_m1_o1_o11_f0_pcu.sym.51.out & UO & 0.46 & 4330 & 2007 & 0.83 & 1.79 & 11.9 & 9.9 & 10.1 & 9.3 & 52.5 & 48.1 \\
\hline str_m1_o1_o11_f0_pcu.sym.71.out & UO & 0.45 & 4436 & 1980 & 0.84 & 1.87 & 13.0 & 10.9 & 10.4 & 9.7 & 52.5 & 48.1 \\
\hline str_m1_o1_o11_f0_pcu.sym.89.out & UO & 0.58 & 3507 & 2043 & 0.83 & 1.42 & 12.4 & 9.8 & 8.2 & 7.7 & 52.5 & 48.1 \\
\hline
\end{tabular}

and $1.3 \mathrm{~g}-\mathrm{H}_{2} \mathrm{~L}^{-1}$. Although these errors are small, a more rigorous validation of the ML can be achieved with GCMC calculations. Thus, GCMC calculations were performed on a subset of MOFs that ML predicted to exhibit high UV and UG capacities. These MOFs fall within the rectangular regions shown in Figs. 6a,b, and exhibit capacities that meet or exceed $36 \mathrm{~g}-\mathrm{H}_{2} \mathrm{~L}^{-1} \& 7.5 \mathrm{wt} . \%$ for PS conditions and $48 \mathrm{~g}-\mathrm{H}_{2} \mathrm{~L}^{-1} \& 7.5 \mathrm{wt} . \%$ under TPS conditions. In total, 21,700 compounds were re-examined with GCMC based on their ML-predicted PS capacities, and another 7,901 were re-examined for TPS.

Figure $6 \mathrm{c}$ compares ML and GCMC predictions for usable capacities for 21,700 high-capacity MOFs under PS conditions. The strong overlap in the two datasets further highlights the accuracy of the ML models. A total of $8,187 \mathrm{MOF}$ were predicted by GCMC to outperform PCN-610/NU-100 under these conditions. A summary of the 10 highest-capacity MOFs, sorted based on their GCMC capacities, is provided in Table 6. (A more extensive listing is provided in Table S10.) The highest capacity MOFs are all hypothetical compounds: 5 originate from the ToBaCCo database, ${ }^{59}$ two are from the Univ. of Ottawa database, ${ }^{37}$ and the remainder are from the Northwestern $^{36}$ database. These MOFs all exhibit high surface areas (average $=5746$, range $\left.=4346-7835 \mathrm{~m}^{2} \mathrm{~g}^{-1}\right)$ and large void fractions of 0.89 , on average. The range of these property values are consistent with those reported in an earlier study, ${ }^{1,136,137}$ and suggest that maximizing the surface area is an important design guideline for PS operation. The highest capacity MOF, mof_7642, ${ }^{59}$ is predicted to exhibit capacities of 11.1 wt. $\%$ and $40.5 \mathrm{~g}-\mathrm{H}_{2} \mathrm{~L}^{-1}$, surpassing that of PCN-610/NU-100, the record-holder under PS conditions. The crystal structure of mof_7642 is shown in Fig. 7a.

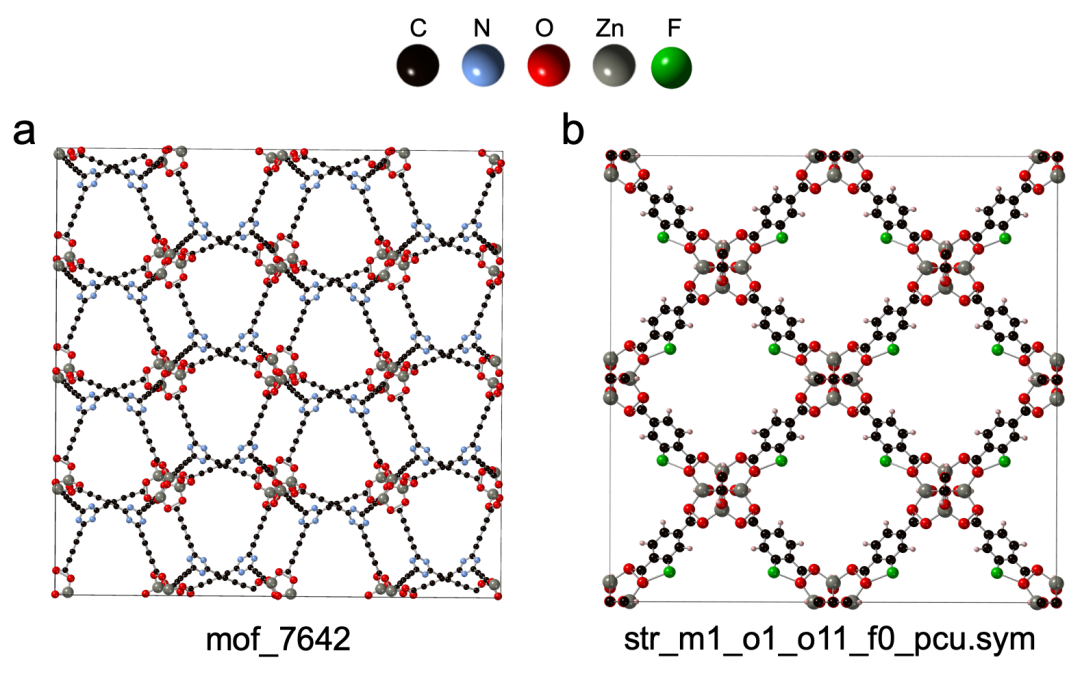

Figure 7. Crystallographic images of the highest-capacity MOFs under (a) pressure swing and (b) temperature swing conditions. These MOFs originate from the $\mathrm{ToBaCCo}^{59}$ and University of Ottawa ${ }^{39}$ databases, respectively. 
Figure 6d provides a similar comparison between ML predictions and GCMC calculations for MOFs expected to exhibit high capacities under TPS conditions. Under these conditions only 95 MOFs were predicted by GCMC to outperform MOF-5. A summary of the 10 highest-capacity MOFs, sorted by their GCMC capacities, is provided in Table 6. (See Table S11 for a more extensive tabulation.) As found for PS operation, all of the top performing candidates are hypothetical compounds. One difference with the PS case is that all of these MOFs originate from the Univ. of Ottawa database. ${ }^{37}$ Furthermore, none of the highest capacity MOFs identified for PS operation appear as top candidates for TPS. Comparing the highest-capacity MOFs for both operating conditions, it can be seen that the high-capacity TPS MOFs systematically exhibit lower surface areas (avg. $\left.=4073 \mathrm{~m}^{2} \mathrm{~g}^{-1}\right)$, smaller void fractions $($ avg. $=0.83)$, and higher densities. Hence, the categories of MOFs that maximize uptake under PS and TPS conditions exhibit distinct properties. These differences suggest that maximizing the surface area - which, as discussed above, is desirable for maximizing PS capacity - is not advantageous for TPS operation. This behavior can be explained by trends in total capacities, ${ }^{6}$ which the TPS capacities reported here approximate. More specifically, it is known that total volumetric capacities are maximized for intermediate values of the surface area; for larger surface areas the volumetric capacity decreases.

Returning to the list of promising MOFs for TPS operation, Table 6 reports that the highest-capacity MOF, str_m1_o1_o11_f0_pcu.sym.102.out, has a GCMC-predicted capacity of 10.4 wt.\% and $53.1 \mathrm{~g}-\mathrm{H}_{2} \mathrm{~L}^{-1}$. This capacity surpasses that of MOF-5, which to our knowledge holds the capacity record under these conditions. The crystal structure of this MOF is shown in Fig. $7 \mathrm{~b}$.

Figures S7-S8 and Table S12 quantify the differences between ML and GCMC predictions on the subset of high-capacity MOFs shown in Figs. 6c,d. For PS operation, the AUE of ML relative to GCMC is 0.24 wt. $\%$ and $0.66 \mathrm{~g}-\mathrm{H}_{2} \mathrm{~L}^{-1}$, while for TPS the AUE is 0.24 wt.\% and $1.28 \mathrm{~g}-\mathrm{H}_{2} \mathrm{~L}^{-1}$. Both sets of errors are comparable to the errors reported in Table 4 for the original test set of MOFs. Figures $S 7(c, f)$ and S8 $(c, f)$ plot the frequency distribution of the differences between GCMC and ML. These distribution plots suggest that the largest differences occur for predictions involving real MOFs and for hypothetical MOFs extracted from databases other than those from Northwestern, ${ }^{36}$ University of Ottawa, ${ }^{37}$ and BJT. ${ }^{83}$ (These MOFs are referred to as "other hypothetical MOFs" in Figure 6). These MOFs, along with the real compounds, exhibit higher structural diversity than those contained in the other databases. For example, the diversity of the topologies used in the $\mathrm{ToBaCCo}^{59}$ and $\mathrm{Zr}-\mathrm{MOFs}^{45}$ databases and in the linkers used in $\mathrm{MTV}-\mathrm{MOF}^{84}$ database are larger than what is found in the databases from Northwestern, ${ }^{36}$ University of Ottawa, ${ }^{37}$ and BJT. ${ }^{83}$

\section{CONCLUSIONS}

The $\mathrm{H}_{2}$ storage capacities of nearly a million MOFs have been predicted via machine learning. The predictions span a diverse collection of MOFs sourced from 19 databases and reveal performance under two operating conditions: pressure swing and temperature + pressure swing. More than a dozen ML algorithms were benchmarked, with the extremely randomized trees method found to be the most accurate. The resulting ML models are accessible on the web at the HyMARC Data Hub. ${ }^{86}$ These models allow for accurate, rapid screening of the hydrogen storage properties of new MOFs using minimal structural data as input; only a single feature is needed for the simplest models.

The accuracy of the ML models was characterized as a function of training set size and the number/combination of input features. Regarding the dependence on the training set, the accuracy of the models can be well-described using a simple power-law function of the training set size. The dependence on the number and combination of input features was determined by evaluating 508 independent $\mathrm{ML}$ models generated from all possible combinations of the seven features. The single most important features for predicting $\mathrm{H}_{2}$ uptake are pore volume (for gravimetric capacity) and void fraction (for volumetric capacity).

Using these models, 8,282 MOFs are identified that have the potential to exceed the capacities of state-of-the-art materials under usable conditions. The identified MOFs are predominantly hypothetical compounds, which (for pressure-swing operation) exhibit low densities $\left(<0.31 \mathrm{~g} \mathrm{~cm}^{-3}\right)$ in combination with high surface areas $(>$ $\left.5,300 \mathrm{~m}^{2} \mathrm{~g}^{-1}\right)$, void fractions $(\sim 0.90)$, and pore volumes $\left(>3.3 \mathrm{~cm}^{3}\right.$ $\left.\mathrm{g}^{-1}\right)$. These MOFs are suggested as targets for experimental synthesis.

\section{AUTHOR INFORMATION}

\section{Corresponding Author}

djsiege@umich.edu; tel: 734-764-4808

\section{Supporting Information}

Document S1. Tables S1-S12, Figures S1-S8, Metrics for ML accuracy. Data S1. Feature_Combination_ML_Models.xlsx

\section{AUTHOR CONTRIBUTIONS}

A.A. conducted the computational components of the project. All authors contributed to the drafting of the paper and to the project idea.

\section{DECLARATION OF INTERESTS}

The authors declare no competing interests.

ACKNOWLEDGMENT. Financial support for this study was provided by the US Department of Energy, Office of Energy Efficiency and Renewable Energy, Grant no. DE-EE0007046. Partial computing resources were provided by the NSF via grant $1531752 \mathrm{MRI}$ : Acquisition of Conflux, A Novel Platform for Data-Driven Computational Physics (Tech. Monitor: Ed Walker). The authors acknowledge Jesse Adams, Dr. Zeric Hulvey, Ms. Courtney Pailing, Mr. Nick Wunder, Ms. Nalinrat Guba and Dr. Caleb Phillips for facilitating web hosting of the ML models. A.A. acknowledges Profs. Randall Snurr and Tom Woo for providing access to their MOF databases; Dr. Maciej Haranczyk for use of the Zeo++ code and the mail-order MOF database; and Prof. Adam J. Matzger, Dr. Antek G. Wong-Foy, Dr. Saona Seth, and Dr. Yiyang Liu for support for MOF design.

\section{REFERENCES}

1.Ahmed, A., Seth, S., Purewal, J., Wong-Foy, A.G., Veenstra, M., Matzger, A.J., and Siegel, D.J. (2019). Exceptional hydrogen storage achieved by screening nearly half a million metal-organic frameworks. Nat. Commun. 10.

2.Ahmed, A., Liu, Y., Purewal, J., Tran, L.D., Veenstra, M., Wong-Foy, A., Matzger, A., and Siegel, D. (2017). Balancing Gravimetric and Volumetric Hydrogen Density in MOFs. Energy Environ. Sci. 10, 2459-2471. 
3.Wong-Foy, A.G., Matzger, A.J., and Yaghi, O.M. (2006). Exceptional H2 Saturation Uptake in Microporous Metal-Organic Frameworks. J. Am. Chem. Soc. 128, 3494-3495.

4.Satyapal, S., Petrovic, J., Read, C., Thomas, G., and Ordaz, G. (2007). The U.S. Department of Energy's National Hydrogen Storage Project: Progress towards meeting hydrogen-powered vehicle requirements. Catal. Today $120,246-256$

5.Greene, D.L., Joseck, F., Duleep, G., and -Oak, G. XI-13 FY 2013 Annual Progress Report DOE Hydrogen and Fuel Cells Program DOE Manager Subcontractor: Overall Objectives Contribution to Achievement of DOE Systems Analysis Milestones XI.1 Worldwide Status of Hydrogen Fuel Cell Vehicle Technology and Prospects for Commercialization XI-14 DOE Hydrogen and Fuel Cells Program FY 2013 Annual Progress Report.

6.Allendorf, M.D., Hulvey, Z., Gennett, T., Ahmed, A., Autrey, T., Camp, J., Seon Cho, E., Furukawa, H., Haranczyk, M., Head-Gordon, M., et al. (2018). An assessment of strategies for the development of solid-state adsorbents for vehicular hydrogen storage. Energy Environ. Sci. 11, 27842812.

7.Yang, J., Sudik, A., Wolverton, C., and Siegel, D.J. (2010). High capacity hydrogen storage materials: attributes for automotive applications and techniques for materials discovery. Chem. Soc. Rev. 39, 656-675.

8.Long, J.R. (2015). 201 "Hydrogen Storage in Metal-Organic Frameworks," J. R. Long; U.S. Department of Energy, Hydrogen and Fuel Cells Program 2015 Annual Merit Review Proceedings: Project ST103. https://www.hydrogen.energy.gov/pdfs/review15/st103_long_2015_o.p df.

9.DOE Technical Targets for Onboard Hydrogen Storage for Light-Duty Vehicles, https://energy.gov/eere/fuelcells/doe-technical-targetsonboard-hydrogen-storage-light-duty-vehicles.

10.Astiaso Garcia, D., Barbanera, F., Cumo, F., Di Matteo, U., and Nastasi, B. (2016). Expert Opinion Analysis on Renewable Hydrogen Storage Systems Potential in Europe. Energies 9, 963.

11.Riis, T., Sandrock, G., Ulleberg, Ø., and Vie, P.J.S. (2006). Hydrogen Storage R\&D: Priorities and Gaps. In Hydrogen Production and Storage: R\&D Priorities and Gaps (International Energy Agency), pp. 19-33.

12.Purewal, J., Veenstra, M., Tamburello, D., Ahmed, A., Matzger, A.J., Wong-Foy, A.G., Seth, S., Liu, Y., and Siegel, D.J. (2019). Estimation of system-level hydrogen storage for metal-organic frameworks with high volumetric storage density. Int. J. Hydrogen Energy 44.

13.Manoharan, Y., Hosseini, S.E., Butler, B., Alzhahrani, H., Senior, B.T.F., Ashuri, T., Krohn, J., Manoharan, Y., Hosseini, S.E., Butler, B., et al. (2019). Hydrogen Fuel Cell Vehicles; Current Status and Future Prospect. Appl. Sci. 9, 2296.

14.Makridis, S.S. (2016). Hydrogen storage and compression. In Methane and Hydrogen for Energy Storage, R. Carriveau and D. S.-K. Ting, eds. (The Institution of Engineering and Technology), pp. 1-28.

15."Ford/BASF-SE/UM Activities in Support of the Hydrogen Storage Engineering Center of Excellence," Mike Veenstra, Jun Yang, Chunchuan Xu, Manuela Gaab, Lena Arnold, Ulrich Muller, Donald J. Siegel, and Yang Ming. (U.S. Department of Energy, Hydrogen and Fuel Cells Program 2014 Annual Merit Review Proceedings).

16.Öhrström, L. (2015). Let's Talk about MOFs-Topology and Terminology of Metal-Organic Frameworks and Why We Need Them. Crystals 5, 154-162.

17.Fischer, R.A., and Schwedler, I. (2014). Terminologie von Metallorganischen Gerüstverbindungen und Koordinationspolymeren (IUPACEmpfehlungen 2013). Angew. Chemie 126, 7209-7214.

18.Batten, S.R., Champness, N.R., Chen, X.-M., Garcia-Martinez, J., Kitagawa, S., Öhrström, L., O’Keeffe, M., Paik Suh, M., and Reedijk, J. (2013). Terminology of metal-organic frameworks and coordination polymers (IUPAC Recommendations 2013). Pure Appl. Chem. 85, 17151724.

19.Thommes, M., Kaneko, K., Neimark, A. V., Olivier, J.P., RodriguezReinoso, F., Rouquerol, J., and Sing, K.S.W. (2015). Physisorption of gases, with special reference to the evaluation of surface area and pore size distribution (IUPAC Technical Report). Pure Appl. Chem. 87, 1051-1069.
20.Batten, S.R., Champness, N.R., Chen, X.-M., Garcia-Martinez, J., Kitagawa, S., Öhrström, L., O’Keeffe, M., Suh, M.P., and Reedijk, J. (2012). Coordination polymers, metal-organic frameworks and the need for terminology guidelines. CrystEngComm 14, 3001.

21.O'Keeffe, M. (2014). Nets, tiles, and metal-organic frameworks. APL Mater. 2, 124106.

22.Tranchemontagne, D.J., Mendoza-Cortés, J.L., O’Keeffe, M., and Yaghi, O.M. (2009). Secondary building units, nets and bonding in the chemistry of metal-organic frameworks. Chem. Soc. Rev. 38, 1257.

23.Reymond, J.-L. (2015). The Chemical Space Project. Acc. Chem. Res. $48,722-730$.

24.Kontijevskis, A. (2017). Mapping of Drug-like Chemical Universe with Reduced Complexity Molecular Frameworks. J. Chem. Inf. Model. 57, 680-699.

25.Martin, R.L., Smit, B., and Haranczyk, M. (2012). Addressing Challenges of Identifying Geometrically Diverse Sets of Crystalline Porous Materials. J. Chem. Inf. Model. 52, 308-318.

26.Sun, D., Sun, F., Deng, X., and Li, Z. (2015). Mixed-Metal Strategy on Metal-Organic Frameworks (MOFs) for Functionalities Expansion: Co Substitution Induces Aerobic Oxidation of Cyclohexene over Inactive $\mathrm{Ni}$ MOF-74. Inorg. Chem. 54, 8639-8643.

27.Deng, H., Doonan, C.J., Furukawa, H., Ferreira, R.B., Towne, J., Knobler, C.B., Wang, B., and Yaghi, O.M. (2010). Multiple functional groups of varying ratios in metal-organic frameworks. Science 327, 846-50.

28.Park, J., Kim, H., Han, S.S., and Jung, Y. (2012). Tuning MetalOrganic Frameworks with Open-Metal Sites and Its Origin for Enhancing $\mathrm{CO}_{2}$ Affinity by Metal Substitution. J. Phys. Chem. Lett. 3, 826-829.

29.Moghadam, P.Z., Li, A., Wiggin, S.B., Tao, A., Maloney, A.G.P., Wood, P.A., Ward, S.C., and Fairen-Jimenez, D. (2017). Development of a Cambridge Structural Database Subset: A Collection of Metal-Organic Frameworks for Past, Present, and Future. Chem. Mater. 29, 2618-2625.

30.Groom, C.R., Bruno, I.J., Lightfoot, M.P., and Ward, S.C. (2016). The Cambridge Structural Database. Acta Crystallogr. Sect. B Struct. Sci. Cryst. Eng. Mater. 72, 171-179

31.Goldsmith, J., Wong-Foy, A.G., Cafarella, M.J., and Siegel, D.J. (2013). Theoretical Limits of Hydrogen Storage in Metal-Organic Frameworks: Opportunities and Trade-Offs. Chem. Mater. 25, 3373-3382.

32.Altintas, C., Avci, G., Daglar, H., Nemati Vesali Azar, A., Erucar, I., Velioglu, S., and Keskin, S. (2019). An extensive comparative analysis of two MOF databases: high-throughput screening of computation-ready MOFs for $\mathrm{CH} 4$ and $\mathrm{H} 2$ adsorption. J. Mater. Chem. A 7, 9593-9608.

33.Chung, Y.G., Camp, J., Haranczyk, M., Sikora, B.J., Bury, W., Krungleviciute, V., Yildirim, T., Farha, O.K., Sholl, D.S., and Snurr, R.Q (2014). Computation-Ready, Experimental Metal-Organic Frameworks: A Tool To Enable High-Throughput Screening of Nanoporous Crystals. Chem. Mater. 26, 6185-6192.

34.Chung, Y.G., Haldoupis, E., Bucior, B.J., Haranczyk, M., Lee, S., Zhang, H., Vogiatzis, K.D., Milisavljevic, M., Ling, S., Camp, J.S., et al. (2019). Advances, Updates, and Analytics for the Computation-Ready, Experimental Metal-Organic Framework Database: CoRE MOF 2019. J. Chem. Eng. Data 64, 5985-5998.

35.Chen, T., and Manz, T.A. (2020). Identifying misbonded atoms in the 2019 CoRE metal-organic framework database. RSC Adv. 10, 2694426951.

36.Wilmer, C.E., Leaf, M., Lee, C.Y., Farha, O.K., Hauser, B.G., Hupp, J.T., and Snurr, R.Q. (2011). Large-scale screening of hypothetical metalorganic frameworks. Nat. Chem. 4, 83-89.

37.Aghaji, M.Z., Fernandez, M., Boyd, P.G., Daff, T.D., and Woo, T.K. (2016). Quantitative Structure - Property Relationship Models for Recognizing Metal Organic Frameworks ( MOFs ) with High CO 2 Working Capacity and $\mathrm{CO} 2 / \mathrm{CH} 4$ Selectivity for Methane Purification. 4505-4511.

38.Martin, R.L., Lin, L.C., Jariwala, K., Smit, B., and Haranczyk, M. (2013). Mail-order metal-organic frameworks (MOFs): Designing isoreticular MOF-5 analogues comprising commercially available organic molecules. J. Phys. Chem. C 117, 12159-12167. 
39.Bao, Y., Martin, R.L., Haranczyk, M., and Deem, M.W. (2015). In silico prediction of MOFs with high deliverable capacity or internal surface area. Phys. Chem. Chem. Phys. 17, 11962-11973.

40.Witman, M., Ling, S., Anderson, S., Tong, L., Stylianou, K.C., Slater, B., Smit, B., and Haranczyk, M. (2016). In silico design and screening of hypothetical MOF-74 analogs and their experimental synthesis. Chem. Sci. 7, 6263-6272.

41.Chung, Y.G., Gómez-gualdrón, D.A., Li, P., Leperi, K.T., Deria, P., Zhang, H., Vermeulen, N.A., Stoddart, J.F., You, F., Hupp, J.T., et al. (2016). In silico discovery of metal-organic frameworks for precombustion $\mathrm{CO} 2$ capture using a genetic algorithm.

42.Anderson, R., Rodgers, J., Argueta, E., Biong, A., and Go, D.A. (2018). Role of Pore Chemistry and Topology in the CO 2 Capture Capabilities of MOFs: From Molecular Simulation to Machine Learning. Chem. Mater 30, 11.

43.Anderson, G., Schweitzer, B., Anderson, R., and Gómez-Gualdrón, D.A. (2019). Attainable Volumetric Targets for Adsorption-Based Hydrogen Storage in Porous Crystals: Molecular Simulation and Machine Learning. J. Phys. Chem. C 123, 120-130.

44.Anderson, R., and Gómez-Gualdrón, D.A. (2019). Increasing topological diversity during computational "synthesis" of porous crystals: how and why. CrystEngComm 21, 1653-1665.

45.Gomez-Gualdron, D.A., Gutov, O. V., Krungleviciute, V., Borah, B., Mondloch, J.E., Hupp, J.T., Yildirim, T., Farha, O.K., and Snurr, R.Q. (2014). Computational Design of Metal-Organic Frameworks Based on Stable Zirconium Building Units for Storage and Delivery of Methane. Chem. Mater. 26, 5632-5639.

46.Bao, Y., Martin, R.L., Simon, C.M., Haranczyk, M., Smit, B., and Deem, M.W. (2015). In Silico Discovery of High Deliverable Capacity Metal-Organic Frameworks. J. Phys. Chem. C 119, 186-195.

47.Willems, T.F., Rycroft, C.H., Kazi, M., Meza, J.C., and Haranczyk, M. (2012). Algorithms and tools for high-throughput geometry-based analysis of crystalline porous materials. Microporous Mesoporous Mater. 149, 134 141.

48.Addicoat, M.A., Coupry, D.E., and Heine, T. (2014). AuToGraFS: Automatic Topological Generator for Framework Structures. J. Phys. Chem. A 118, 9607-9614.

49.Boyd, P.G., and Woo, T.K. (2016). A generalized method for constructing hypothetical nanoporous materials of any net topology from graph theory. CrystEngComm 18, 3777-3792.

50.Gómez-Gualdrón, D.A., Colón, Y.J., Zhang, X., Wang, T.C., Chen, Y.S., Hupp, J.T., Yildirim, T., Farha, O.K., Zhang, J., and Snurr, R.Q. (2016). Evaluating topologically diverse metal-organic frameworks for cryoadsorbed hydrogen storage. Energy Environ. Sci. 9, 3279-3289.

51.Yao, Z., Sanchez-Lengeling, B., Bobbitt, N.S., Bucior, B.J., Kumar, S.G.H., Collins, S.P., Burns, T., Woo, T.K., Farha, O., Snurr, R.Q., et al. (2020). Inverse Design of Nanoporous Crystalline Reticular Materials with Deep Generative Models. https://doi.org/10.26434/chemrxiv.12186681.v2.

52.Sadus, R.J. (1999). Molecular simulation of fluids: theory, algorithms, and object-orientation. (Elsevier)

53.Allen, M.P., and Tildesley, D.J. (1989). Computer simulation of liquids (Oxford University Press).

54.Frenkel, D., and Smit, B. (2001). Understanding molecular simulation : from algorithms to applications 2 nd ed. (Academic Press, Inc.).

55.Hill, T.L. (1986). An introduction to statistical thermodynamics (Dover Publications).

56.Dubbeldam, D., Torres-Knoop, A., and Walton, K.S. (2013). Molecular Simulation On the inner workings of Monte Carlo codes On the inner workings of Monte Carlo codes. Mol. Simul. 39, 14-15.

57.Fernandez, M., Boyd, P.G., Daff, T.D., Aghaji, M.Z., and Woo, T.K. (2014). Rapid and Accurate Machine Learning Recognition of High Performing Metal Organic Frameworks for CO 2 Capture. J. Phys. Chem. Lett. 5, 3056-3060.
58.Martin, R.L., Simon, C.M., Smit, B., and Haranczyk, M. (2014). In silico Design of Porous Polymer Networks: High-Throughput Screening for Methane Storage Materials. J. Am. Chem. Soc. 136, 5006-5022.

59.Colón, Y.J., Gómez-Gualdrón, D.A., and Snurr, R.Q. (2017). Topologically Guided, Automated Construction of Metal-Organic Frameworks and Their Evaluation for Energy-Related Applications. Cryst. Growth Des. 17, 5801-5810.

60.Boyd, P.G., Moosavi, S.M., Witman, M., and Smit, B. (2017). ForceField Prediction of Materials Properties in Metal-Organic Frameworks. J. Phys. Chem. Lett. 8, 357-363.

61.Thornton, A.W., Simon, C.M., Kim, J., Kwon, O., Deeg, K.S., Konstas, K., Pas, S.J., Hill, M.R., Winkler, D.A., Haranczyk, M., et al. (2017). Materials Genome in Action: Identifying the Performance Limits of Physical Hydrogen Storage. Chem. Mater. 29, 2844-2854.

62.Bobbitt, N.S., and Snurr, R.Q. (2019). Molecular Simulation Molecular modelling and machine learning for high-throughput screening of metal-organic frameworks for hydrogen storage Molecular modelling and machine learning for high-throughput screening of metal-organic frameworks for hydrogen storage.

63.Borboudakis, G., Stergiannakos, T., Frysali, M., Klontzas, E., Tsamardinos, I., and Froudakis, G.E. (2017). Chemically intuited, largescale screening of MOFs by machine learning techniques. npj Comput. Mater. 3.

64.Broom, D.P., Webb, C.J., Hurst, K.E., Parilla, P.A., Gennett, T., Brown, C.M., Zacharia, R., Tylianakis, E., Klontzas, E., Froudakis, G.E., et al. (2016). Outlook and challenges for hydrogen storage in nanoporous materials. Appl. Phys. A 122, 151.

65.Butler, K.T., Davies, D.W., Cartwright, H., Isayev, O., and Walsh, A. (2018). Machine learning for molecular and materials science. Nature 559, $547-555$

66.Wahiduzzaman, M., Walther, C.F.J., and Heine, T. (2014). Hydrogen adsorption in metal-organic frameworks: The role of nuclear quantum effects. J. Chem. Phys. 141, 064708.

67.Durette, D., Bénard, P., Zacharia, R., and Chahine, R. (2016). Investigation of the hydrogen adsorbed density inside the pores of MOF-5 from path integral grand canonical Monte Carlo at supercritical and subcritical temperature. Sci. Bull. 61, 594-600.

68.Fischer, M., Hoffmann, F., and Fröba, M. (2009). Preferred hydrogen adsorption sites in various MOFs-A comparative computational study. ChemPhysChem 10, 2647-2657.

69.Furukawa, H., Miller, M.A., and Yaghi, O.M. (2007). Independent verification of the saturation hydrogen uptake in MOF-177 and establishment of a benchmark for hydrogen adsorption in metal-organic frameworks. J. Mater. Chem. 17, 3197.

70.Fischer, M., Hoffmann, F., and Fröba, M. (2009). Preferred hydrogen adsorption sites in various MOFs-A comparative computational study. ChemPhysChem 10, 2647-2657.

71.Ahmed, A., and Siegel, D.J. (2019). HyMARC datahub. https://datahub.hymarc.org/dataset/computational-prediction-ofhydrogen-storage-capacities-in-mofs. https://datahub.hymarc.org/; https://datahub.hymarc.org/dataset/computational-prediction-ofhydrogen-storage-capacities-in-mofs

72.Pardakhti, M., Moharreri, E., Wanik, D., Suib, S.L., and Srivastava, R. (2017). Machine Learning Using Combined Structural and Chemical Descriptors for Prediction of Methane Adsorption Performance of Metal Organic Frameworks (MOFs). ACS Comb. Sci. 19, 640-645.

73.Fernandez, M., Woo, T.K., Wilmer, C.E., and Snurr, R.Q. (2013). Large-Scale Quantitative Structure-Property Relationship (QSPR) Analysis of Methane Storage in Metal-Organic Frameworks. J. Phys. Chem. C 117, 7681-7689.

74.Fernandez, M., Trefiak, N.R., and Woo, T.K. (2013). Atomic Property Weighted Radial Distribution Functions Descriptors of Metal-Organic Frameworks for the Prediction of Gas Uptake Capacity. J. Phys. Chem. C $117,14095-14105$ 
75.Fernandez, M., and Barnard, A.S. (2016). Geometrical Properties Can Predict $\mathrm{CO}_{2}$ and $\mathrm{N}_{2}$ Adsorption Performance of Metal-Organic Frameworks (MOFs) at Low Pressure. ACS Comb. Sci. 18, 243-252.

76.Nanoporous Materials Genome Center http://www.chem.umn.edu/nmgc/.

77.Hastie, T., Tibshirani, R., and Friedman, J. (2009). The Elements of Statistical Learning (Springer New York).

78.Thornton, A.W., Simon, C.M., Kim, J., Kwon, O., Deeg, K.S., Konstas, K., Pas, S.J., Hill, M.R., Winkler, D.A., Haranczyk, M., et al. (2017). Materials Genome in Action: Identifying the Performance Limits of Physical Hydrogen Storage. Chem. Mater. 29, 2844-2854.

79.Dorugade, A. V, and Kashid, D.N. (2010). Alternative Method for Choosing Ridge Parameter for Regression.

80.Van Wieringen, W.N. (2020). Lecture notes on ridge regression.

81.Smola, A.J., Smola, A.J., and Schölkopf, B. (2004). A tutorial on support vector regression.

82.Bucior, B.J., Bobbitt, N.S., Islamoglu, T., Goswami, S., Gopalan, A., Yildirim, T., Farha, O.K., Bagheri, N., and Snurr, R.Q. Energy-based descriptors to rapidly predict hydrogen storage in metal-organic frameworks. Mol. Syst. Des. Eng. 2018. DOI 10.1039/c8me00050f.

83.Lan, Y., Yan, T., Tong, M., and Zhong, C. (2019). Large-scale computational assembly of ionic liquid/MOF composites: Synergistic effect in the wire-tube conformation for efficient $\mathrm{CO} 2 / \mathrm{CH} 4$ separation. J. Mater. Chem. A 7, 12556-12564.

84.Li, S., Chung, Y.G., Simon, C.M., and Snurr, R.Q. (2017). HighThroughput Computational Screening of Multivariate Metal- Organic Frameworks (MTV-MOFs) for CO 2 Capture. J. Phys. Chem. Lett 8, 19.

85. Geurts, P., Ernst, D., and Wehenkel, L. (2006). Extremely randomized trees. Mach. Learn. 63, 3-42.

86.Ahmed, A., and Siegel, D.J. HyMARC Sorbent Machine Learning Model: Predicting the hydrogen storage capacity of metal-organic frameworks via machine learning. https://sorbent-ml.hymarc.org/.

87.Willems, T.F., Rycroft, C.H., Kazi, M., Meza, J.C., and Haranczyk, M. (2012). Algorithms and tools for high-throughput geometry-based analysis of crystalline porous materials. Microporous Mesoporous Mater. 149, 134141.

88.Pinheiro, M., Martin, R.L., Rycroft, C.H., Jones, A., Iglesia, E., and Haranczyk, M. (2013). Characterization and comparison of pore landscapes in crystalline porous materials. J. Mol. Graph. Model. 44, 208-219.

89.Pinheiro, M., Martin, R.L., Rycroft, C.H., and Haranczyk, M. (2013). High accuracy geometric analysis of crystalline porous materials. CrystEngComm 15, 7531-7538.

90. Ongari, D., Boyd, P.G., Barthel, S., Witman, M., Haranczyk, M., and Smit, B. (2017). Accurate Characterization of the Pore Volume in Microporous Crystalline Materials.

91.Boyd, P.G., Chidambaram, A., García-Díez, E., Ireland, C.P., Daff, T.D., Bounds, R., Gładysiak, A., Schouwink, P., Moosavi, S.M., MarotoValer, M.M., et al. (2019). Data-driven design of metal-organic frameworks for wet flue gas $\mathrm{CO} 2$ capture. Nature 576, 253-256.

92.Boyd, P.G., Chidambaram, A., García-Díez, E., Ireland, C.P., Daff, T.D., Bounds, R., Gładysiak, A., Schouwink, P., Moosavi, S.M., MarotoValer, M.M., et al. (2019). Data-driven design of metal-organic frameworks for wet flue gas CO2 capture, Materials Cloud Archive 2018.0016/v3 (2019), doi: 10.24435/materialscloud:2018.0016/v3. Nature 576, 253256.

93.R-WLLFHS: https://github.com/snurr-group/Reduced-hMOFdatabase.

94.Wolpert, D.H., and Macready, W.G. (1997). No Free Lunch Theorems for Optimization. IEEE Trans. Evol. Comput. 1.

95.Breiman, L., Friedman, J.H., Olshen, R.A., and Stone, C.J. (2017). Classification And Regression Trees (Routledge).

96.Freund, Y., and Schapire, R.E. (1997). A Decision-Theoretic Generalization of On-Line Learning and an Application to Boosting. J. Comput. Syst. Sci. 55, 119-139.
97.Drucker, H. (1997). Improving Regressors using Boosting Techniques. ICML '97 Proc. Fourteenth Int. Conf. Mach. Learn., 107-115. https://dl.acm.org/doi/10.5555/645526.657132.

98.Breiman, L. (1996). Bagging Predictors. Mach. Learn. 24, 123-140.

99.Breiman, L. (2001). Random forests. Mach. Learn. 45, 5-32.

100.Friedman, J. (2001). Greedy Function Approximation: A Gradient Boosting Machine. Ann. Stat. 29, 1189-1232.

101.Chang, C.-C., and Lin, C.-J. (2001). LIBSVM: A Library for Support Vector Machines.

102.Platt, J.C., and Platt, J.C. (1999). Probabilistic Outputs for Support Vector Machines and Comparisons to Regularized Likelihood Methods. Adv. LARGE MARGIN Classif., 61--74.

103.Buhmann, M.D. (2002). Radial Basis Functions: Theory and Implementations (Cambridge University Press).

104.Fan, R.-E., Chang, K.-W., Hsieh, C.-J., Wang, X.-R., and Lin, C.-J. (2008). LIBLINEAR: A Library for Large Linear Classification.

105. Rifkin, R.M.., and Lippert, R.A. (2007). Notes on Regularized Least Squares.

106.Altman, N.S. (1992). An introduction to kernel and nearestneighbor nonparametric regression. Am. Stat. 46, 175-185.

107.Freund, Y., and Schapire, R.E. (1999). A Short Introduction to Boosting. J. Japanese Soc. Artif. Intell. 14, 771-780.

108.Fernández-Delgado, M., Sirsat, M.S., Cernadas, E., Alawadi, S., Barro, S., and Febrero-Bande, M. (2019). An extensive experimental survey of regression methods. Neural Networks 111, 11-34.

109.Pedregosa, F., Varoquaux, G., Gramfort, A., Michel, V., Thirion, B., Grisel, O., Blondel, M., Prettenhofer, P., Weiss, R., Dubourg, V., et al. (2011). Scikit-learn: Machine Learning in Python. J. Mach. Learn. Res. 12, 2825-2830.

110. Richard, M.-A., Bénard, P., and Chahine, R. (2009). Gas adsorption process in activated carbon over a wide temperature range above the critical point. Part 1: modified Dubinin-Astakhov model. Adsorption 15, 43-51.

111.Gomez-Gualdron, D.A., Wang, T.C., García-Holley, P., Sawelewa, R.M., Argueta, E., Snurr, R.Q., Hupp, J.T., Yildirim, T., and Farha, O.K. (2017). Understanding Volumetric and Gravimetric Hydrogen Adsorption Trade-off in Metal-Organic Frameworks. ACS Appl. Mater. Interfaces 9, 33419-33428.

112.García-Holley, P., Schweitzer, B., Islamoglu, T., Liu, Y., Lin, L., Rodriguez, S., Weston, M.H., Hupp, J.T., Gómez-Gualdrón, D.A., Yildirim, T., et al. (2018). Benchmark Study of Hydrogen Storage in Metal-Organic Frameworks under Temperature and Pressure Swing Conditions. ACS Energy Lett., 748-754.

113.Düren, T., Bae, Y.-S., and Snurr, R.Q. (2009). Using molecular simulation to characterise metal-organic frameworks for adsorption applications. Chem. Soc. Rev. 38, 1237.

114.Allendorf, M.D., Bauer, C.A., Bhakta, R.K., and Houk, R.J.T. (2009) Luminescent metal-organic frameworks. Chem. Soc. Rev. 38, 1330.

115.Gómez-Gualdrón, D.A., Moghadam, P.Z., Hupp, J.T., Farha, O.K., and Snurr, R.Q. (2016). Application of Consistency Criteria To Calculate BET Areas of Micro- And Mesoporous Metal-Organic Frameworks. J. Am. Chem. Soc. 138, 215-224.

116. Himanen, L., Geurts, A., Foster, A.S., and Rinke, P. (2019). DataDriven Materials Science: Status, Challenges, and Perspectives. Adv. Sci., 1900808 .

117.Wei, J., Chu, X., Sun, X., Xu, K., Deng, H., Chen, J., Wei, Z., and Lei, M. (2019). Machine learning in materials science. InfoMat 1, 338-358.

118.Fanourgakis, G.S., Gkagkas, K., Tylianakis, E., Klontzas, E., and Froudakis, G. (2019). A Robust Machine Learning Algorithm for the Prediction of Methane Adsorption in Nanoporous Materials. J. Phys. Chem. A, acs.jpca.9b03290.

119.Panella, B., Hirscher, M., and Roth, S. (2005). Hydrogen adsorption in different carbon nanostructures. Carbon N. Y. 43, 2209-2214.

120.Kiyabu, S., Lowe, J.S., Ahmed, A., and Siegel, D.J. (2018). Computational Screening of Hydration Reactions for Thermal Energy Storage: New Materials and Design Rules. Chem. Mater. 30, 2006-2017. 
121.Moosavi, S.M., Chidambaram, A., Talirz, L., Haranczyk, M., Stylianou, K.C., and Smit, B. (2019). Capturing chemical intuition in synthesis of metal-organic frameworks. Nat. Commun. 10, 539.

122.Zwillinger, D., Kokoska, S., Raton, B., New, L., and Washington, Y. (2000). standard probability and Statistics tables and formulae CRC.

123. Oliphant, T.E. (2007). Python for Scientific Computing. Comput. Sci. Eng. 9, 10-20.

124.Millman, K.J., and Aivazis, M. (2011). Python for Scientists and Engineers. Comput. Sci. Eng. 13, 9-12.

125.Parrt, T., and Turgutlu, K. rfpimp 1.3.4, https://github.com/parrt/random-forest-importances.

126.Machine, P., and With, L. (2017). Improve Machine Learning Results with Boosting, Bagging and Blending Ensemble Methods in Weka Start and Practice Machine Learning With Weka. 1-12.

127.Here, S., Products, B., and Contact, A. (2017). Classification And Regression Trees for Machine Learning. 1-11.

128.Witman, M., Ling, S., Grant, D.M., Walker, G.S., Agarwal, S., Stavila, V., and Allendorf, M.D. (2020). Extracting an Empirical Intermetallic Hydride Design Principle from Limited Data via Interpretable Machine Learning. J. Phys. Chem. Lett. 11, 40-47.

129.Sturluson, A., Huynh, M.T., Kaija, A.R., Laird, C., Yoon, S., Hou, F., Feng, Z., Wilmer, C.E., Colón, Y.J., Chung, Y.G., et al. (2019). The role of molecular modelling and simulation in the discovery and deployment of metal-organic frameworks for gas storage and separation. Mol. Simul. 45, 1082-1121.

130.Barthel, S., Alexandrov, E. V, Proserpio, D.M., and Smit, B. (2018). Distinguishing Metal-Organic Frameworks.

131.Altintas, C., Avci, G., Daglar, H., Nemati Vesali Azar, A., Erucar, I., Velioglu, S., and Keskin, S. (2019). An extensive comparative analysis of two MOF databases: high-throughput screening of computation-ready MOFs for $\mathrm{CH}_{4}$ and $\mathrm{H}_{2}$ adsorption. J. Mater. Chem. A 7, 9593-9608.

132. Chen, T., and Manz, T.A. (2019). A collection of forcefield precursors for metal-organic frameworks. RSC Adv. 9, 36492-36507.

133.Panella, B., Hirscher, M., and Roth, S. (2005). Hydrogen adsorption in different carbon nanostructures. Carbon N. Y. 43, 2209-2214.

134. Sarkisov, L., Bueno-Perez, R., Sutharson, M., and Fairen-jimenez, D. (2020). Material Informatics with PoreBlazer v4.0 and CSD MOF Database.

135.Chen, Z., Li, P., Anderson, R., Wang, X., Zhang, X., Robison, L., Redfern, L.R., Moribe, S., Islamoglu, T., Gómez-Gualdrón, D.A., et al. (2020). Balancing volumetric and gravimetric uptake in highly porous materials for clean energy. Science (80-. ). 368, 297-303.

136.Camp, J.S., Stavila, V., Allendorf, M.D., Prendergast, D., and Haranczyk, M. (2018). Critical Factors in Computational Characterization of Hydrogen Storage in Metal-Organic Frameworks Critical Factors in Computational Characterization of Hydrogen Storage in Metal-Organic Frameworks.

137.Churchard, A.J., Banach, E., Borgschulte, A., Caputo, R., Chen, J.C., Clary, D., Fijalkowski, K.J., Geerlings, H., Genova, R. V., Grochala, W., et al. (2011). A multifaceted approach to hydrogen storage. Phys. Chem. Chem. Phys. 13, 16955-16972. 